\title{
QUEEN'S
UNIVERSITY
BELFAST
}

\section{Chemical processes in protoplanetary disks. II. On the importance of photochemistry and X-ray ionization}

Walsh, C., Nomura, H., Millar, TJ., \& Aikawa, Y. (2012). Chemical processes in protoplanetary disks. II. On the importance of photochemistry and X-ray ionization. Astrophysical Journal, 747(114).

https://doi.org/10.1088/0004-637X/747/2/114

Published in:

Astrophysical Journal

Document Version:

Publisher's PDF, also known as Version of record

Queen's University Belfast - Research Portal:

Link to publication record in Queen's University Belfast Research Portal

Publisher rights

Copyright 2012. The American Astronomical Society. This work is made available online in accordance with the publisher's policies. Please refer to any applicable terms of use of the publisher.

\section{General rights}

Copyright for the publications made accessible via the Queen's University Belfast Research Portal is retained by the author(s) and / or other copyright owners and it is a condition of accessing these publications that users recognise and abide by the legal requirements associated with these rights.

Take down policy

The Research Portal is Queen's institutional repository that provides access to Queen's research output. Every effort has been made to ensure that content in the Research Portal does not infringe any person's rights, or applicable UK laws. If you discover content in the Research Portal that you believe breaches copyright or violates any law, please contact openaccess@qub.ac.uk. 


\title{
CHEMICAL PROCESSES IN PROTOPLANETARY DISKS. II. ON THE IMPORTANCE OF PHOTOCHEMISTRY AND X-RAY IONIZATION
}

\author{
Catherine Walsh $^{1}$, Hideko Nomura $^{2}$, T. J. Millar ${ }^{1}$, and Yuri Aikawa ${ }^{3}$ \\ ${ }^{1}$ Astrophysics Research Centre, School of Mathematics and Physics, Queen's University Belfast, \\ University Road, Belfast BT7 1NN, UK; catherine.walsh@qub.ac.uk \\ ${ }^{2}$ Department of Astronomy, Graduate School of Science, Kyoto University, Kyoto 606-8502, Japan \\ ${ }^{3}$ Department of Earth and Planetary Sciences, Kobe University, 1-1 Rokkodai-cho, Nada, Kobe 657-8501, Japan \\ Received 2011 June 21; accepted 2012 January 12; published 2012 February 22
}

\begin{abstract}
We investigate the impact of photochemistry and X-ray ionization on the molecular composition of, and ionization fraction in, a protoplanetary disk surrounding a typical T Tauri star. We use a sophisticated physical model, which includes a robust treatment of the radiative transfer of UV and X-ray radiation, and calculate the time-dependent chemical structure using a comprehensive chemical network. In previous work, we approximated the photochemistry and X-ray ionization; here, we recalculate the photoreaction rates using the explicit UV wavelength spectrum and wavelength-dependent reaction cross sections. We recalculate the X-ray ionization rate using our explicit elemental composition and X-ray energy spectrum. We find that photochemistry has a larger influence on the molecular composition than X-ray ionization. Observable molecules sensitive to the photorates include $\mathrm{OH}, \mathrm{HCO}^{+}, \mathrm{N}_{2} \mathrm{H}^{+}, \mathrm{H}_{2} \mathrm{O}, \mathrm{CO}_{2}$, and $\mathrm{CH}_{3} \mathrm{OH}$. The only molecule significantly affected by the X-ray ionization is $\mathrm{N}_{2} \mathrm{H}^{+}$, indicating that it is safe to adopt existing approximations of the X-ray ionization rate in typical T Tauri star-disk systems. The recalculation of the photorates increases the abundances of neutral molecules in the outer disk, highlighting the importance of taking into account the shape of the UV spectrum in protoplanetary disks. A recalculation of the photoreaction rates also affects the gas-phase chemistry due to the adjustment of the $\mathrm{H} / \mathrm{H}_{2}$ and $\mathrm{C}^{+} / \mathrm{C}$ ratios. The disk ionization fraction is not significantly affected by the methods adopted to calculate the photochemistry and X-ray ionization. We determine that there is a probable "dead zone" where accretion is suppressed, present in a layer, $Z / R \lesssim 0.1-0.2$, in the disk midplane, within $R \approx 200 \mathrm{AU}$.
\end{abstract}

Key words: astrochemistry - ISM: molecules - protoplanetary disks - stars: formation

Online-only material: color figures

\section{INTRODUCTION}

Protoplanetary disks have several vital functions in star and planet formation: they (1) aid the dissipation of angular momentum away from the protostellar system, (2) allow the efficient accretion of matter from the constituent cloud material onto the central star, and (3) contain the material which may eventually form an accompanying planetary system.

Protoplanetary disks are physically and thus, chemically, complex objects (see, e.g., Bergin et al. 2007). They are heavily irradiated by UV radiation from their parent $\mathrm{T}$ Tauri star and are permeated by X-rays and excess UV photons thought to arise from an accretion shock generated as disk material impinges upon the stellar surface (Herbig \& Goodrich 1986; Kastner et al. 1997). Beyond a radius $r \gtrsim 100 \mathrm{AU}$, the UV radiation field originating from the parent star decreases in strength due to a combination of absorption of UV photons by the intervening disk material and geometrical dilution. Here, irradiation by UV photons originating from the interstellar radiation field (ISRF) increases in importance and, as a result, the wavelength dependence (or shape) of the radiation field varies as a function of disk radius and height (Aikawa \& Herbst 1999; Willacy \& Langer 2000). As a result, in modern disk models, wavelengthdependent radiative transfer is preferred (van Zadelhoff et al. 2003 ) as is the inclusion of UV excess radiation (Bergin et al. 2003; Nomura \& Millar 2005) and X-rays (Glassgold et al. 1997; Aikawa \& Herbst 1999). Along with cosmic rays, the X-ray and UV radiation controls the ionization fraction in the disk which has consequences on the disk accretion rate and the location and extent of "dead zones," regions where accretion is potentially suppressed (Balbus \& Hawley 1991; Gammie 1996). The varying radiation field will also have a direct effect on the disk chemical structure, controlling the abundance and distribution of atoms, ions, and molecules through photochemistry and influencing the molecular composition of the icy grain mantle via non-thermal desorption and this has been demonstrated in many works (see, e.g., van Zadelhoff et al. 2003; Aikawa \& Nomura 2006; Walsh et al. 2010; Kamp et al. 2010; Vasyunin et al. 2011). For these reasons, the treatment of photo processes in protoplanetary disks should be thoroughly investigated, in order to aid the interpretation of observational data, especially with the impending completion of the Atacama Large Millimeter Array (ALMA) which, for the first time, will enable the observation of molecular emission from nearby $(\sim 140 \mathrm{pc})$ protoplanetary disks on around submilliarcsecond scales with unprecedented spectral resolution.

A plethora of molecules have been detected in protoplanetary disks via line emission in the (sub)millimeter and infrared regions of the electromagnetic spectrum. Early observations at (sub)millimeter wavelengths were made using the James Clerk Maxwell Telescope and IRAM $30 \mathrm{~m}$ radio telescope (e.g., Kastner et al. 1997; Dutrey et al. 1997; van Zadelhoff et al. 2001; Thi et al. 2004), with more recent detections using the Submillimeter Array (SMA; e.g., Qi et al. 2006, 2008; Öberg et al. 2010). Most molecules observed in this spectral region are small, simple, abundant molecules, molecular ions, and radicals (e.g., $\mathrm{CO}, \mathrm{CN}, \mathrm{CS}, \mathrm{HCO}^{+}, \mathrm{N}_{2} \mathrm{H}^{+}, \mathrm{HCN}$ ) and associated isotopologues (e.g., ${ }^{13} \mathrm{CO}, \mathrm{DCO}^{+}$, and $\mathrm{C}^{34} \mathrm{~S}$ ). A recent survey of disks around $\mathrm{T}$ Tauri and Herbig Ae stars has led to the first successful detection of SO in a circumstellar disk (Fuente et al. 2010), with 
the authors also reporting a tentative detection of $\mathrm{H}_{2} \mathrm{~S}$. Due to the limitations of existing telescopes and the small angular size of disks, the most complex molecule observed to date is formaldehyde, $\mathrm{H}_{2} \mathrm{CO}$ (Dutrey et al. 1997; Aikawa et al. 2003).

Use of the Infrared Spectrograph (IRS) on the Spitzer Space Telescope increased the inventory of gas-phase molecules detected in disks to include $\mathrm{OH}, \mathrm{H}_{2} \mathrm{O}, \mathrm{CO}_{2}$, and $\mathrm{C}_{2} \mathrm{H}_{2}$ (Lahuis et al. 2006; Carr \& Najita 2008; Salyk et al. 2008; Pontoppidan et al. 2010). Existing (sub)millimeter observations probe the colder, outer disk material whereas infrared observations probe the warmer gas in the inner disk surface in the socalled planet-forming region. There have also been detections of water ice absorption features in "edge-on" $\mathrm{T}$ Tauri systems (Creech-Eakman et al. 2002; Terada et al. 2007; Schegerer \& Wolf 2010). More recently, Hogerheijde et al. (2011) report the first detection of the ground-state rotational emission lines of both spin isomer states of water in a protoplanetary disk using the Heterodyne Instrument for the Far-Infrared (HIFI) mounted on the Herschel Space Observatory. These sets of observations, of both gas and ice, give us a reasonably sufficient benchmark with which we can compare our results.

In Walsh et al. (2010), henceforth referred to as Paper I, we used the physical disk model described in Nomura \& Millar (2005) and Nomura et al. (2007) to compute the chemical structure of a typical protoplanetary disk on small scales (submilliarcsecond in the inner disk for an object at the distance of Taurus, $\sim 140 \mathrm{pc}$ ), investigating the effects of the addition of non-thermal desorption mechanisms (cosmic-ray-induced desorption, photodesorption, and X-ray desorption) and grain-surface chemistry on the disk chemical structure. In that work, we presented results from models in which we approximated the photoreaction rates by scaling the rates from the UMIST Database for Astrochemistry or UDfA (Woodall et al. 2007), which assume the ISRF, by the wavelength-integrated UV flux at each point in the disk (see Section 2.2.1 for further details). Here, we report results from models in which we explicitly calculate the photodissociation and photoionization rates taking into consideration the UV spectrum at each point and the wavelength-dependent absorption cross section for each photoreaction. In addition, we recalculate the X-ray ionization rate everywhere in the disk accounting for the elemental composition of the gas and include the direct X-ray ionization of elements, in both cases, using the $\mathrm{X}$-ray energy spectrum at each point.

In Section 2.1, we give a brief overview of our physical model. In Section 2.2, we describe our chemical network and processes we include in our calculation of the chemical structure with a thorough description of the methods used to compute the photochemical and X-ray ionization rates (Sections 2.2.1 and 2.2.2, respectively). In Section 2.3, we describe the theory behind the identification of regions of our disk in which angular momentum transport and thus, accretion, may be suppressed. The results of our calculations are presented in Section 3 with a summary given in Section 4.

\section{PROTOPLANETARY DISK MODEL}

\subsection{Physical Model}

The physical model of a protoplanetary disk we use is from Nomura \& Millar (2005) with the addition of X-ray heating as described in Nomura et al. (2007). The degree of ionization in the disk depends on the disk parameters adopted and the resulting surface density distribution. The theoretical foundation of our model comes from the standard accretion disk model of
Lynden-Bell \& Pringle (1974) and Pringle (1981) which defines a surface density distribution for the disk given the central star's mass and radius and a disk accretion rate, $\dot{M}$. The kinematic viscosity in the disk is parameterized according to the work of Shakura \& Sunyaev (1973), the so-called, $\alpha$-prescription. We consider an axisymmetric disk surrounding a typical T Tauri star with mass, $M_{*}=0.5 M_{\odot}$, radius, $R_{*}=2 R_{\odot}$, and effective temperature, $T_{*}=4000 \mathrm{~K}$. We adopt a viscous parameter, $\alpha=$ 0.01 and a mass accretion rate, $\dot{M}=10^{-8} M_{\odot} \mathrm{yr}^{-1}$.

We use a model X-ray spectrum created by fitting the observed XMM-Newton spectrum of the classical T Tauri star, TW Hydrae (Kastner et al. 2002) with a two-temperature thin thermal plasma model (MEKAL model; see, e.g., Liedahl et al. 1995). The derived best-fit parameters for the plasma temperatures are $k T_{1}=0.8 \mathrm{keV}$ and $k T_{2}=0.2 \mathrm{keV}$ and for the foreground interstellar hydrogen column density, $N_{\mathrm{H}}=$ $2.7 \times 10^{20} \mathrm{~cm}^{-2}$. For the $\mathrm{X}$-ray extinction, we include attenuation due to ionization of all elements and Compton scattering by hydrogen. The X-ray luminosity is $L_{\mathrm{X}} \sim 10^{30} \mathrm{erg} \mathrm{s}^{-1}$ and the resulting high-resolution $X$-ray spectrum is given in Figure 1 of Nomura et al. (2007) assuming a distance to source of $56 \mathrm{pc}$, and is reproduced in binned form here in the right-hand panel of Figure 1.

The UV radiation field in disks has two sources, the star and the interstellar medium. In this disk model, the radiation field due to the T Tauri star has three components: blackbody emission at the star's effective temperature, optically thin hydrogenic bremsstrahlung emission, and strong Ly $\alpha$ line emission. For the UV extinction, we include absorption and scattering by dust grains. We assume that the dust and gas in the disk are well mixed and adopt a dust-size distribution model which reproduces the observational extinction curve of dense clouds (Weingartner \& Draine 2001). The calculation of the dust opacity in the disk is described in Appendix D of Nomura \& Millar (2005) with the resulting wavelength-dependent absorption coefficient shown in Figure D.1. The total far-UV luminosity in our model is $L_{\mathrm{UV}} \sim 10^{31} \mathrm{erg} \mathrm{s}^{-1}$ with the calculation of the radiation field in the disk described in detail in Appendix C of Nomura \& Millar (2005). We display the resulting stellar flux density in the disk surface at a radius of $1 \mathrm{AU}$, including each individual component, in the left-hand panel of Figure 1. The main source of UV photons shortward of $2000 \AA$ is due to bremsstrahlung and $\operatorname{Ly} \alpha$ radiation with the $\operatorname{Ly} \alpha$ line contributing around $10^{3}$ times the UV continuum photon flux at $\approx 1216 \AA$ over an assumed FWHM of $\approx 2 \AA$ (see, e.g., Herczeg et al. 2002).

The resulting disk physical structure is given in Figures 1 and 13 in Paper I and we refer readers to the Appendix of that publication for a thorough discussion. In Figure 2, we display the gas and dust temperatures in Kelvin (top right), the gas number density in $\mathrm{cm}^{-3}$ (top left), the wavelength-integrated UV flux (bottom left), and X-ray flux (bottom right) both in units of erg $\mathrm{cm}^{-2} \mathrm{~s}^{-1}$, as a function of disk radius and height (scaled by the radius). In the temperature plot, the color map represents the gas temperature whereas the contours represent the dust temperature. As expected, the disk surface closest to the parent star is subjected to the largest flux of both UV and $\mathrm{X}$-ray radiation. The disk midplane is effectively completely shielded from UV photons over the radial extent of our disk model. The higher energy X-ray photons, although resulting in a lower flux in the disk surface, are able to penetrate the disk more effectively, leading to a small, yet appreciable, X-ray flux in the disk midplane beyond $\approx 10 \mathrm{AU}$. 

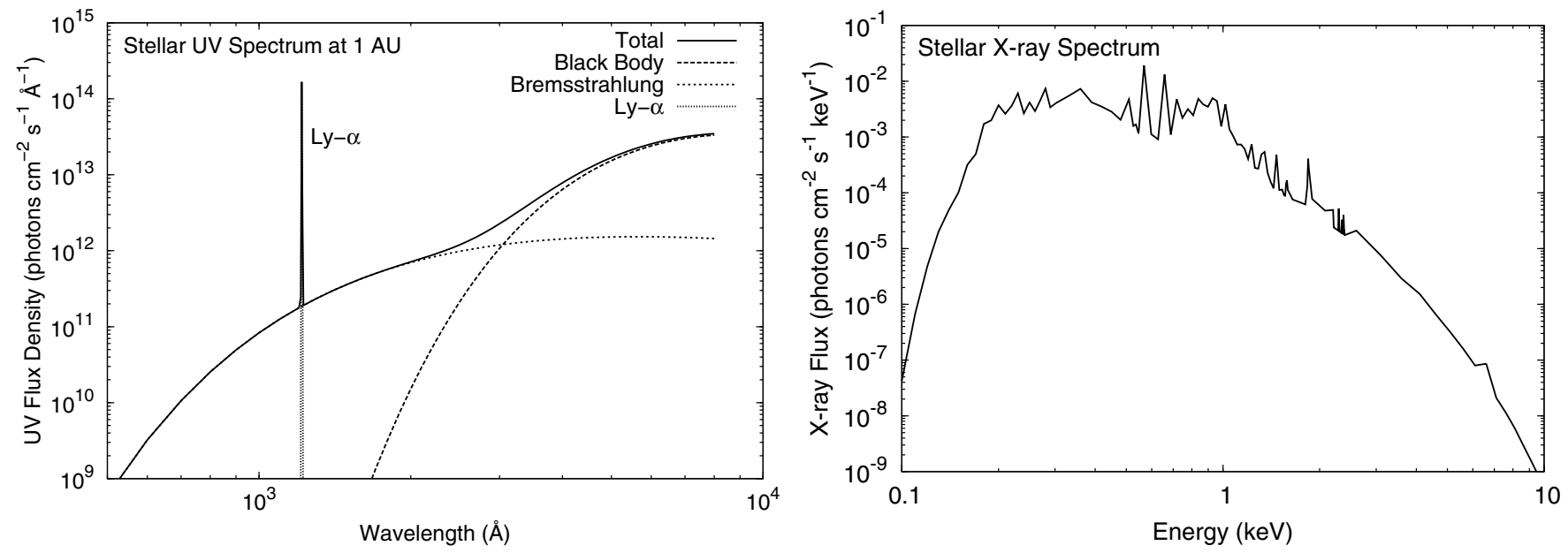

Figure 1. Stellar UV flux density in the disk surface at $1 \mathrm{AU}$ in photons $\mathrm{cm}^{-2} \mathrm{~s}^{-1} \AA^{-1}$ (left) and binned X-ray flux density in photons $\mathrm{cm}^{-2} \mathrm{~s}^{-1} \mathrm{keV}^{-1}$ (right). The latter assumes a distance to source of $56 \mathrm{pc}$.
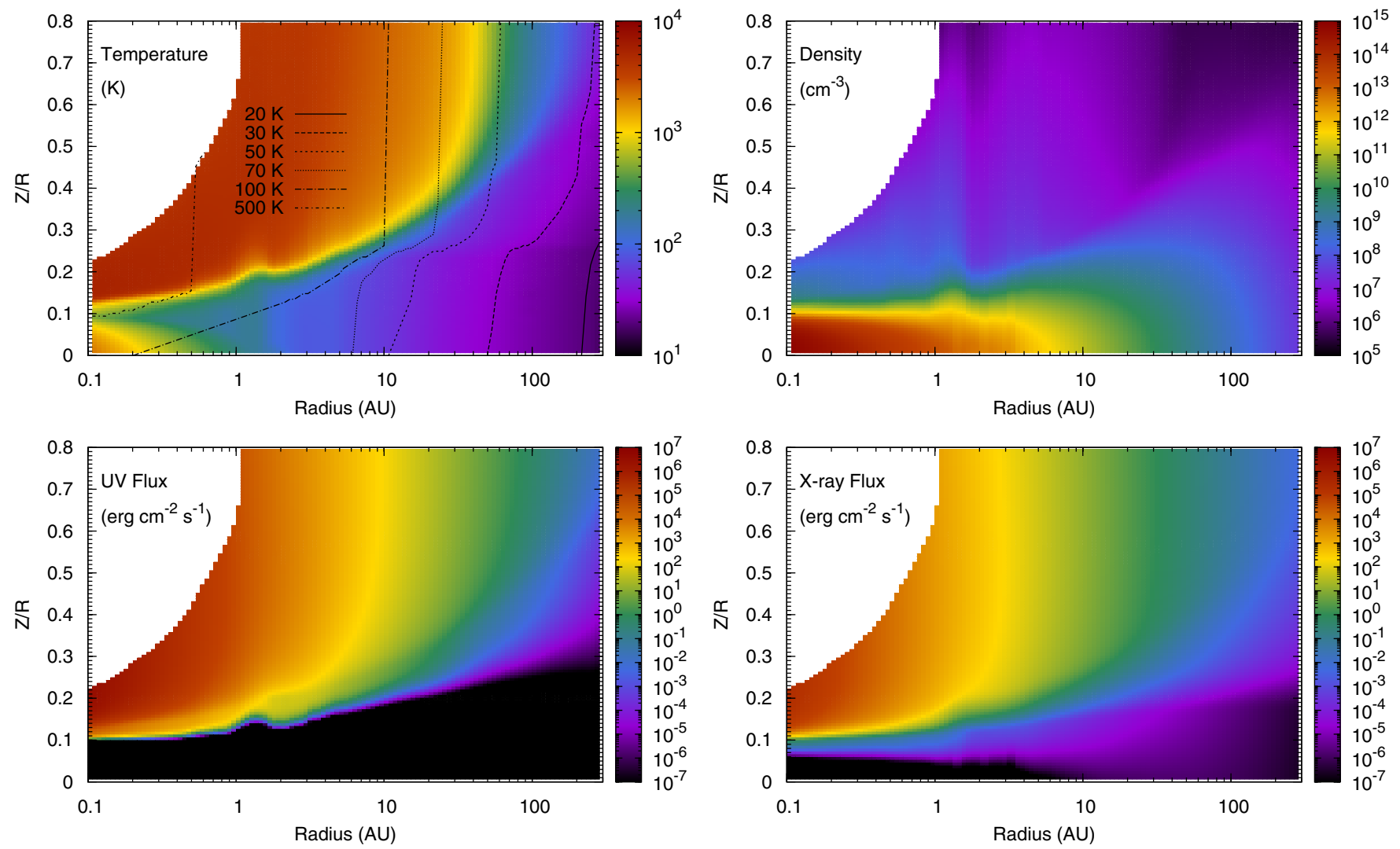

Figure 2. Temperature (top left), number density (top right), UV flux (bottom left), and X-ray flux (bottom right) as a function of disk radius, $R$, and height (scaled by the radius, i.e., $Z / R$ ). On the temperature panel, the color map represents the gas temperature whereas the contours represent the dust temperature.

(A color version of this figure is available in the online journal.)

\subsection{Chemical Model}

As in Paper I, our gas-phase chemistry is extracted from the latest release of the UDfA or "Rate06," available at http://www.udfa.net (Woodall et al. 2007). We use almost the entire Rate06 network removing only those reactions involving fluorine- and phosphorus-containing species. Here, we also include the subset of three-body reactions from Rate06, since these may play a significant role in the densest regions of our disk model $\left(n \sim 10^{15} \mathrm{~cm}^{-3}\right)$.

We allow gas-grain interactions, i.e., the accretion of gasphase species onto dust grains with removal of the grain mantle via thermal desorption, cosmic-ray-induced desorption (Léger et al. 1985; Hasegawa \& Herbst 1993), and photodesorption (Westley et al. 1995; Öberg et al. 2007; Willacy 2007). In Paper I we provided a thorough description of the methods used to determine our accretion and desorption rates. We also include the grain-surface network from Hasegawa et al. (1992) and Hasegawa \& Herbst (1993).

Here, we calculate our photodesorption rates taking into account the molecular composition of the grain mantle. Experiments into the photodesorption of UV-irradiated ices conducted by Öberg et al. (2009b, 2009c) suggest that the photodesorption yields are dependent on the ice composition with pure CO, 
Table 1

Photodesorption Yields

\begin{tabular}{lcc}
\hline \hline Species & $\begin{array}{c}\text { Yield } \\
(\text { molecules photon }\end{array}$ & Reference \\
\hline $\mathrm{CO}$ & $2.7 \times 10^{-3}$ & 1 \\
$\mathrm{~N}_{2}$ & $1.8 \times 10^{-4}$ & 1 \\
$\mathrm{CO}_{2}$ & $2.3 \times 10^{-3}$ & 2 \\
$\mathrm{H}_{2} \mathrm{O}$ & $1.3 \times 10^{-3}$ & 2 \\
All other species & $3.0 \times 10^{-3}$ & 3 \\
\hline
\end{tabular}

References. (1) Öberg et al. 2009c; (2) Öberg et al. 2009b; (3) Westley et al. 1995.

$\mathrm{N}_{2}, \mathrm{CO}_{2}$, and $\mathrm{H}_{2} \mathrm{O}$ ices giving different desorption yields (listed in Table 1). The picture is further complicated by evidence of codesorption in mixed ices, with the photodesorption yield of $\mathrm{N}_{2}$ increasing when present in a $1: 1 \mathrm{~N}_{2} / \mathrm{CO}$ ice mixture (Öberg et al. 2007). More recent work suggests that in organic ices, irradiation by UV photons initiates chemistry with photodesorption products detected other than those present in the original ice mixture (Öberg et al. 2009a). These latter effects are not included in this model for simplicity; however, they should be investigated in future models.

In Paper I, we used a constant photodesorption yield of $3 \times 10^{-3}$ photon $^{-1}$ as determined for pure water ice by Westley et al. (1995). To take into consideration the composition of the grain mantle we now determine the photodesorption rate for a specific species according to

$$
k_{\mathrm{pd}}^{i}=G_{\mathrm{UV}} Y_{\mathrm{UV}}^{i} \sigma_{\mathrm{d}} x_{\mathrm{d}} x_{s}^{i} \mathrm{~s}^{-1},
$$

where $G_{\mathrm{UV}}$ is the radiation field in units of photons $\mathrm{cm}^{-2} \mathrm{~s}^{-1}$, $Y_{\mathrm{UV}}^{i}$ photon $^{-1}$ is the specific desorption yield of species $i$, as listed in Table $1, x_{\mathrm{s}}^{i}=n_{\mathrm{s}}^{i} / n_{\mathrm{s}}^{\text {tot }}$ is the fractional abundance of species $i$ on the dust grains and $\sigma_{\mathrm{d}}$ and $x_{\mathrm{d}}$ are the dust-grain geometrical cross section and fractional abundance, respectively. We have also reviewed our set of molecular desorption energies in light of recent experimental results and in Table 2 we list those species for which the binding energies, $E_{\mathrm{d}}$, have been updated.

Our initial fractional abundances are the result of a dark cloud model run with typical molecular cloud parameters, i.e., $T=10 \mathrm{~K}, n\left(\mathrm{H}_{2}\right)=10^{4} \mathrm{~cm}^{-3}$, and $A_{v}=10 \mathrm{mag}$ using the set of oxygen-rich low-metallicity elemental abundances from Graedel et al. (1982) as listed in Table 8 of Woodall et al. (2007). In the generation of our initial abundances we allow for freezeout and thermal desorption and we extract abundances at a time of $10^{5} \mathrm{yr}$, which is thought to be the age of dark clouds on the brink of star formation.

\subsubsection{Photochemistry}

The photoreaction rates in Rate06 are calculated assuming the UV radiation field is given by the Draine field (Draine 1978), an adequate assumption for the unshielded ISRF. For use in chemical models, these rates are subsequently parameterized according to optical depth or $A_{v}$. In Paper I, we approximated our photoreaction rates in the disk, $k_{\mathrm{ph}}$, by scaling the rates from Rate06 using the wavelength-integrated UV flux at each point,

$$
\begin{gathered}
G_{\mathrm{UV}}(r, z)=\int_{912 \AA}^{2000 \AA} G_{\lambda}(r, z) d \lambda, \text { i.e., } \\
k_{\mathrm{ph}}=\frac{G_{\mathrm{UV}}}{G_{0}} k_{0} \mathrm{~s}^{-1},
\end{gathered}
$$

where $G_{0}$ is the unshielded interstellar UV flux and $k_{0}$ is the rate calculated for the unshielded interstellar medium. Note that
Table 2

Molecular Binding Energies

\begin{tabular}{lcc}
\hline \hline Species & $\begin{array}{c}\text { Binding Energy } \\
(\mathrm{K})\end{array}$ & Reference \\
\hline $\mathrm{CO}$ & 855 & 1 \\
$\mathrm{~N}_{2}$ & 790 & 1 \\
$\mathrm{CO}_{2}$ & 2990 & 2 \\
$\mathrm{C}_{2} \mathrm{~S}$ & 5320 & 2 \\
$\mathrm{H}_{2} \mathrm{O}$ & 4800 & 3 \\
$\mathrm{SO}_{2}$ & 5330 & 2 \\
$\mathrm{NH}_{3}$ & 2790 & 3 \\
$\mathrm{CH}_{4}$ & 1090 & 4 \\
$\mathrm{HCOOH}_{\mathrm{CH}} \mathrm{OH}$ & 5000 & 5 \\
$\mathrm{CH}_{3} \mathrm{CHO}$ & 4930 & 3 \\
$\mathrm{C}_{2} \mathrm{H}_{6}$ & 3800 & 5 \\
$\mathrm{HCOOCH}_{3}$ & 2300 & 5 \\
$\mathrm{CH}_{3} \mathrm{OCH}$ & 4000 & 5 \\
$\mathrm{C}_{2} \mathrm{H}_{5} \mathrm{OH}$ & 3300 & 5 \\
\hline
\end{tabular}

References. (1) Öberg et al. 2005; (2) Edridge 2010; (3) Brown \& Bolina 2007; (4) Herrero et al. 2010; (5) Öberg et al. 2009a.

$G_{\lambda}(r, z)$ includes both the stellar and interstellar components of the radiation field. This approximation is unsuitable for use in protoplanetary disks as these objects are irradiated by UV radiation with three components: the central star, UV excess due to accretion and the ISRF. Hence, the UV spectrum at each point in the disk will not only vary with disk radius and height, but will also bear no resemblance to the ISRF (see Figure 4 in Nomura \& Millar 2005). For these reasons, we have investigated a recalculation of the photorates in the disk taking into consideration the UV spectrum at each point (van Dishoeck 1987; van Dishoeck et al. 2006).

The photodissociation rate due to continuous absorption, $k_{\mathrm{ph}}^{\mathrm{c}}$, is calculated using

$$
k_{\mathrm{ph}}^{\mathrm{c}}=\int_{\lambda_{\min }}^{\lambda_{\max }} \sigma(\lambda) I(\lambda) d \lambda \mathrm{s}^{-1},
$$

where $\lambda$ is the wavelength, $\sigma(\lambda)$ is the cross section, and $I(\lambda)$ is the mean intensity of UV radiation. The rate for photoionization, $k_{\mathrm{pi}}$, is calculated using the same equation. For indirect photodissociation via absorption into a bound upper state, $u$, from a lower state, $l$, the rate is

$$
k_{\mathrm{ph}}^{1}=\frac{\pi e^{2}}{m c^{2}} \lambda_{\mathrm{ul}}^{2} f_{\mathrm{ul}} \mu_{u} I(\lambda) \mathrm{s}^{-1},
$$

where $\lambda_{\mathrm{ul}}$ is the wavelength of the line transition, $f_{\mathrm{ul}}$ is the oscillator strength of the transition, and $\mu_{\mathrm{ul}}$ is an efficiency factor. The parameters, $e, c$, and $m$ are the electron charge, the speed of light, and the atomic or molecular mass, respectively. The total photodissociation rate is found by summing over all possible channels. The photoreaction cross sections, $\sigma(\lambda)$, are those adopted in van Zadelhoff et al. (2003) which originate from calculations by van Dishoeck (1988), updated by Jansen et al. (1995a, 1995b) and van Dishoeck et al. (2006). The photo cross sections are downloadable from http://www.strw.leidenuniv.nl/ ewine/photo/. For species which do not have a calculated cross section, we use the rate for a similar type of molecule.

We should note here that we include $\operatorname{Ly} \alpha$ radiation in the calculation of the wavelength-integrated UV flux (Equation (2)) which accounts for approximately $85 \%$ of the total flux (see, 
e.g., Bergin et al. 2003); however, we ignore it in the calculation of the wavelength-dependent UV radiation field and subsequent photorates (see Equations (3) and (4)). The exclusion of Ly $\alpha$ is primarily due to the difficulties in treating the scattering of Ly $\alpha$ photons from the surface into the disk (see, e.g., Bergin et al. 2003). Ly $\alpha$ scattering differs from that of the background UV photons since the scattering occurs predominantly by $\mathrm{H}$ atoms rather than dust grains. Indeed, Ly $\alpha$ radiation has been historically neglected in protoplanetary disk models and has only very recently been addressed in the work by Bethell \& Bergin (2011). However, the photorates calculated according to Equations (3) and (4) are more accurate than those determined using Equation (2) since the shape of the background radiation field is included in the calculation. The photorates calculated using Equation (2) include the total flux of UV photons (background plus Ly $\alpha$ ) heavily overestimating the strength of the UV field at wavelengths other than the Ly $\alpha$ wavelength $(\approx 1216 \AA)$. The effect of including the wavelength dependence in the photorates is apparent in differences in the relative abundances of molecules (see Table 4), e.g., the column density ratio of $\mathrm{CO}_{2} / \mathrm{H}_{2} \mathrm{O}$ changes from $\approx 0.045$ in model UV-old to $\approx 1.90$ in model UV-new at $100 \mathrm{AU}$. We discuss the possible effects of including $\operatorname{Ly} \alpha$ radiation in the calculation of the photorates in Section 3.6.

We include the self-shielding of $\mathrm{H}_{2}$ using the approximation from Federman et al. (1979) in the generation of our physical model giving us the initial conditions in our disk; however, we do not explicitly include the self- and mutual shielding of $\mathrm{H}_{2}$ and $\mathrm{CO}$ in our calculation of the subsequent chemical structure. We find that the dominant component of the radiation field in the disk surface is the radial component which is the direct stellar radiation (the vertical component consists of both the contribution from the ISRF and scattered stellar radiation). In addition, throughout the majority of the disk, the stellar radiation dominates over the interstellar radiation. Hence, we argue against the validity of adopting the usual plane-parallel approximation for the calculation of the self-shielding factors and the application of shielding factors computed for irradiated interstellar clouds, to protoplanetary disks. To correctly include the effects of self- and mutual shielding in disks, a selfconsistent two-dimensional treatment is needed which takes into consideration the time-varying $\mathrm{H}_{2}$ and $\mathrm{CO}$ abundances throughout the disk, the column densities in the radial and vertical direction and the two-dimensional physical structure of the disk which will effect the line widths and line strengths and hence, shielding factors. We discuss this issue further in Section 3.5.

\subsubsection{X-Ray Ionization}

The model of Nomura et al. (2007) calculates an overall X-ray ionization rate at each point in the disk according to the theory of Maloney et al. (1996). The rate at each point is calculated assuming a power-law fit for the X-ray absorption cross section dependent on X-ray energy and it is these approximate rates that are used in our chemical calculations in Paper I. In this work, we recalculate the $\mathrm{X}$-ray ionization rate everywhere in the disk taking into account the X-ray energy spectrum, $F_{\mathrm{X}}(E)$, at each point and the explicit elemental composition of the gas (Glassgold et al. 1997). The overall X-ray ionization rate, $\zeta_{\mathrm{XR}}$, is given by summing over all elements,

$$
\zeta_{\mathrm{XR}}=\sum_{k} \int_{E_{k}}^{E_{\max }} x_{k} \sigma_{k}(E) F_{\mathrm{X}}(E)\left[\frac{E-E_{k}}{\Delta \epsilon}\right] d E \mathrm{~s}^{-1},
$$

where, for each element, $k, E_{k}$ is the ionization potential, $\sigma_{k}(E)$ is the cross section, and $x_{k}$ is the fractional abundance with respect to $\mathrm{H}$ nuclei density. In this expression, the number of secondary ionizations per unit energy produced by primary photoelectrons, $N_{\mathrm{sec}}$, is given by the expression, $\left(E-E_{k}\right) / \Delta \epsilon$, where $\Delta \epsilon$ is the mean energy required to make an ion pair $(\approx 37 \mathrm{eV})$. Only the number of secondary ionizations needs to be considered as this is generally much larger than the number of primary ionization events. Typically, each keV of secondary electron energy produces an average of $1000 / 37 \approx 27$ ion pairs so that $N_{\text {sec }} \gg N_{\text {pri }}$. Note that X-rays interact only with atoms, regardless of whether an atom is bound within a molecule or free (Glassgold et al. 1997).

We have also added the direct X-ray ionization of elements, the rate for which is given by

$$
\zeta_{k}=\int_{E_{k}}^{E_{\max }} \sigma_{k}(E) F_{\mathrm{X}}(E) d E \mathrm{~s}^{-1}
$$

using the ionization cross sections for each element, $k$, from Verner et al. (1993).

\subsection{Disk Ionization Fraction}

In addition to investigating the importance of photochemistry and X-ray chemistry in protoplanetary disks, we have determined the location and extent of potential dead zones where accretion may be inhibited. Angular momentum transport in disks is thought to arise from turbulence initiated by a weakfield magnetorotational instability or MRI (Balbus \& Hawley 1991); hence, accretion may be inefficient in regions where the instability is suppressed. The turbulence generated by the instability can sustain a disordered magnetic field to which the gas is coupled. The degree of the coupling, in turn, depends on the ionization fraction in the disk.

Following Gammie (1996), we can define a magnetic Reynolds number, $\operatorname{Re}_{\mathrm{M}}$, everywhere,

$$
\operatorname{Re}_{\mathrm{M}}=\frac{V_{\mathrm{A}} H}{\eta}
$$

where $V_{\mathrm{A}} \approx \alpha^{1 / 2} c_{s}$ is the Alfvén speed, a function of $\alpha$, the scaling parameter for the viscosity $\left(v=\alpha c_{s} H\right)$ from the accretion disk model of Shakura \& Sunyaev (1973), and $c_{s}$ is the sound speed in the disk. $H$ is the disk scale height given by $H=c_{s} / \Omega$, where $\Omega$ is the Keplerian velocity at a particular radius, $R$, and $\eta$ is the magnetic resistivity which is related to the electron fraction, $\chi \equiv n_{\mathrm{e}} / n_{\mathrm{H}}$ by

$$
\eta=\left(6.5 \times 10^{3}\right) \chi^{-1} \mathrm{~cm}^{2} \mathrm{~s}^{-1}
$$

(see Gammie 1996 for further details). Accretion is likely suppressed in regions where the magnetic Reynolds number, $\mathrm{Re}_{\mathrm{M}}$, falls below a critical value, $\operatorname{Re}_{M}^{\text {crit }}$. This parameter determines the degree to which the ionized gas is effectively coupled to the magnetic field. Recent MHD simulations suggest $\operatorname{Re}_{M}^{\text {crit }} \sim 100$ (Sano \& Stone 2002; Ilgner \& Nelson 2006), which corresponds roughly to an electron fraction, $\chi \sim 10^{-12}$, although the exact value is dependent on the disk model adopted. Using our chemical model results, we calculate the value of the magnetic Reynolds number, $\mathrm{Re}_{\mathrm{M}}$, everywhere and identify possible dead zones where $\operatorname{Re}_{\mathrm{M}} \lesssim 100$.

Chiang \& Murray-Clay (2007) argue that a second criterion, taking into account the influence of ambipolar diffusion in 
suppressing the MRI, must be applied for protoplanetary disks. They define a dimensionless parameter, Am, which describes the degree to which neutral $\mathrm{H}_{2}$ molecules (which make up the bulk of the gas), are coupled to the accreting plasma. In order for neutral gas to be unstable, an $\mathrm{H}_{2}$ molecule must collide with enough ions within the $e$-folding time of the instability, $1 / \Omega$, where $\Omega$ is the Keplerian velocity of the gas:

$$
\mathrm{Am}=\frac{x_{\mathrm{e}} n \beta}{\Omega}>\mathrm{Am}^{\mathrm{crit}} .
$$

Here, $x_{\mathrm{e}}$ is the disk ionization fraction, $n$ is the number density of the neutral gas, and $\beta \approx 2.0 \times 10^{-9} \mathrm{~cm}^{3} \mathrm{~s}^{-1}$ is the rate coefficient for ion-neutral collisions. Early MHD simulations by Hawley \& Stone (1998) suggest that sufficient turbulence and angular momentum transport are achieved when the "ambipolar diffusion parameter," Am, exceeds a critical value, Am ${ }^{\text {crit }} \approx$ 100. More recent simulations by Bai \& Stone (2011) suggest that in very weakly ionized media where the "strong coupling" limit holds, such as protoplanetary disks, the critical value for Am is $\approx 1$. The "strong coupling" limit is defined as when the ion inertia is negligible and the recombination time is much shorter than the orbital time (Bai 2011). We adopt this latter criterion in our determination of the susceptibility of the disk to suppression of the MRI by ambipolar diffusion. The results are reported in Section 3.

Note that the extent and location of a dead zone is also dependent on the treatment of gas-grain interactions and the size distribution of grains (Sano et al. 2000; Ilgner \& Nelson 2006). Sano et al. (2000) calculated that, for a fixed gas-to-dust mass ratio, the dead zone shrinks as the grain size increases, assuming that all grains have the same radius. As the grains increase in size (likely due to coagulation) the ion density increases since the total surface area of grains decreases. This subsequently decreases the recombination rate of gas-phase cations on grain surfaces. They also determine that in the midplane, grains are the dominant charged species (with charge $\pm e$ ).

It has also been postulated that gravitational grain settling (or sedimentation) toward the disk midplane influences the protoplanetary disk physical and chemical structure and thus, ionization fraction, the persistence of the MRI and the subsequent location and extent of dead zones (see, e.g., Chiang et al. 2001; Dullemond \& Dominik 2004; D'Alessio et al. 2006; Nomura et al. 2007; Fogel et al. 2011; Vasyunin et al. 2011). Since the dust is the dominant source of opacity in disks, grain settling allows deeper penetration of stellar and interstellar UV radiation potentially ionizing a larger proportion of the gaseous component of the disk. In particular, D'Alessio et al. (2006) find that an absence of small grains in the upper disk layer, due to sedimentation, enhances the ionization in the disk surface and also decreases the temperature of the gas in the disk midplane since there is a decrease in the amount of radiation processed by grains and directed toward the midplane. In reality, disk ionization, turbulence by MRI, and dust settling are coupled and thus ideally should be solved self-consistently (see, e.g., Fromang \& Papaloizou 2006; Ciesla 2007; Turner et al. 2010).

In this work, for our gas-grain interactions, we assume a constant grain radius of $0.1 \mu \mathrm{m}$ and a fixed dust-grain fractional abundance of $2.2 \times 10^{-12}$. We assume that all grains are negatively charged and allow the recombination of cations on grain surfaces. This is valid assumption since negatively charged grains dominate in regions where the number density, $n$, is $\lesssim 10^{12} \mathrm{~cm}^{-3}$ and this holds throughout most of our disk model. We intend to investigate the effects of adding neutral grains and
Table 3

Chemical Models

\begin{tabular}{lcccc}
\hline \hline Chemical Process & UV-old & UV-new & XR+UV-old & XR+UV-new \\
\hline Thermal desorption & $\checkmark$ & $\checkmark$ & $\checkmark$ & $\checkmark$ \\
Cosmic-ray-induced desorption & $\checkmark$ & $\checkmark$ & $\checkmark$ & $\checkmark$ \\
Photodesorption & $\checkmark$ & $\checkmark$ & $\checkmark$ & $\checkmark$ \\
Grain-surface chemistry & $\checkmark$ & $\checkmark$ & $\checkmark$ & $\checkmark$ \\
Photochemistry & & $\checkmark$ & & $\checkmark$ \\
X-ray ionization & & & $\checkmark$ & $\checkmark$ \\
\hline
\end{tabular}

a variable dust-grain size distribution caused by coagulation and settling in future models.

We have also neglected the thermal ionization of alkali metals, such as $\mathrm{Na}^{+}$and $\mathrm{K}^{+}$, which becomes a significant source of ionization when the gas temperature is greater than $\approx 10^{3} \mathrm{~K}$ (Fromang et al. 2002; Ilgner \& Nelson 2006). In our disk model, we find that in the midplane the gas temperature reaches values higher than this only within a radius of a few tenths of an AU; nevertheless, it should be looked at in future models since, due to this source of ionization, the gas may be magnetorotationally unstable close to the star.

\section{RESULTS}

We calculate the chemical abundances in the disk as a function of radius, height, and time. The results displayed in this section are those extracted at a time of $10^{6} \mathrm{yr}$, the typical age of visible $\mathrm{T}$ Tauri stars with accompanying protoplanetary disks. Throughout this section, fractional abundance refers to the abundance of each species with respect to total particle number density. As in Paper I, we ran several different models with differing chemical ingredients in order to determine the influence of each chemical process and these are listed in Table 3. Here, model UV-old is our "fiducial" model in which we use the same method as in Paper I to calculate the photochemical rates and we use the X-ray ionization rates as calculated in Nomura et al. (2007). We compare the results from model UV-old with those from models which include a recalculation of the photochemical rates only (model UV-new), the X-ray ionization rates only (model XR+UV-old), and both processes (model XR+UV-new). All models include freezeout, thermal desorption, cosmic-ray-induced desorption, photodesorption, and grain-surface chemistry.

\subsection{Column Densities}

In Figure 3, we present the radial column density $\left(\mathrm{cm}^{-2}\right)$ of molecules detected or searched for in protoplanetary disks at both (sub)millimeter and infrared wavelengths. The solid lines and dashed lines are the gas-phase and grain-surface column densities, respectively.

Molecules whose column densities are relatively unaffected by the method employed to calculate the photoreaction rates and the X-ray ionization rate include $\mathrm{CO}, \mathrm{CN}, \mathrm{CS}, \mathrm{C}_{2} \mathrm{H}, \mathrm{C}_{2} \mathrm{H}_{2}$, $\mathrm{H}_{2} \mathrm{CO}, \mathrm{HCN}, \mathrm{OH}, \mathrm{CH}_{4}$, and $\mathrm{SO}$. Over the radial extent of the disk, the column densities of the listed species vary, at most, by a factor of a few between chemical models. The gas-phase column densities of $\mathrm{H}_{2} \mathrm{O}$ and $\mathrm{CH}_{3} \mathrm{OH}$ are affected only beyond a radius of $\approx 1 \mathrm{AU}$ with that of $\mathrm{CO}_{2}$ altered beyond a radius of $\approx 10 \mathrm{AU}$. In all three cases we see a rise in the column density of each species when the photorates are recalculated. For the molecular ions, $\mathrm{HCO}^{+}$and $\mathrm{N}_{2} \mathrm{H}^{+}$, we see a different behavior with the column density of the former species affected significantly within a 

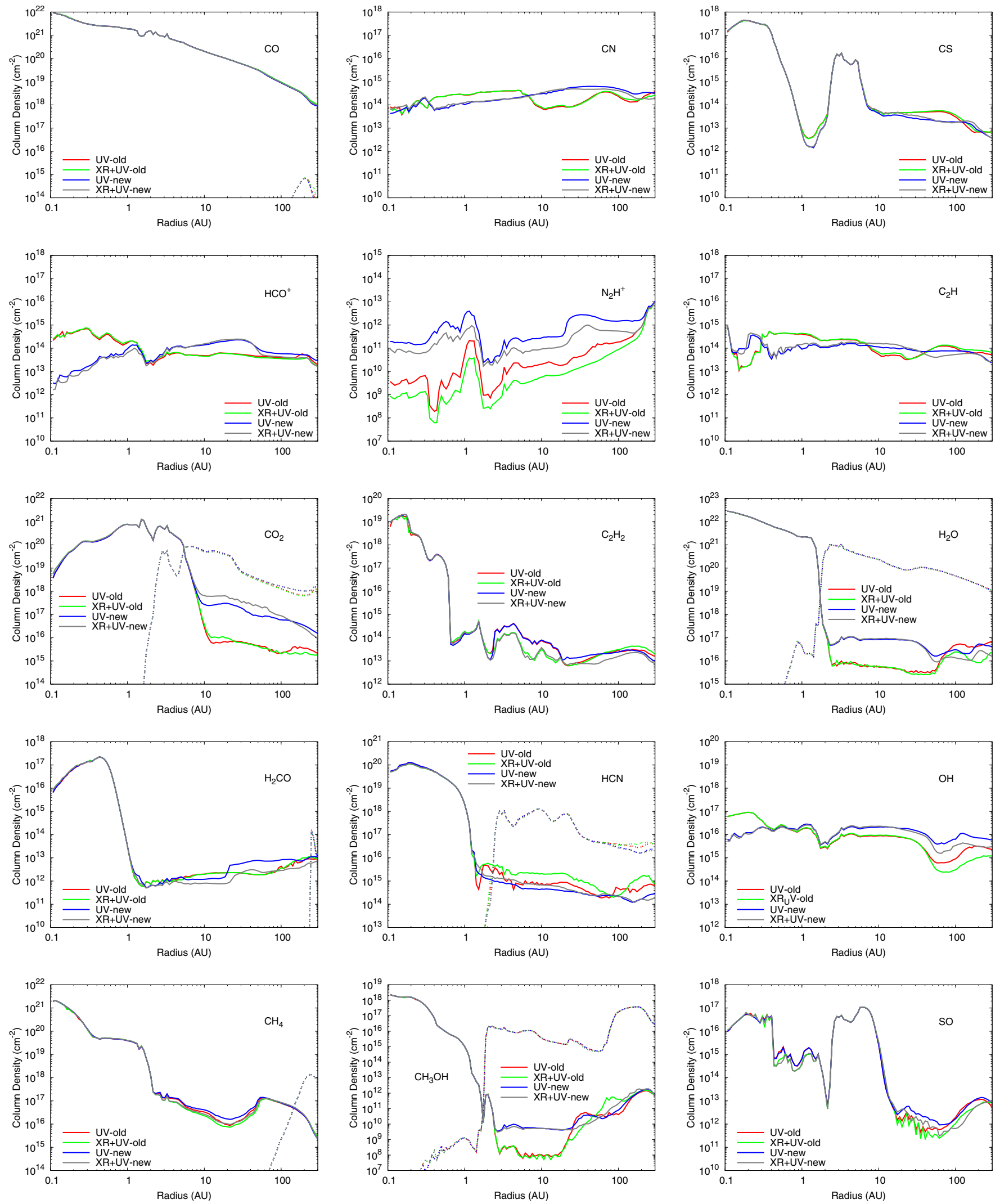

Figure 3. Column density $\left(\mathrm{cm}^{-2}\right)$ as a function of radius, $R$, for a range of molecules detected or searched for in protoplanetary disks. The dashed lines represent grain-surface (ice) column densities.

(A color version of this figure is available in the online journal.) 
radius of approximately $1 \mathrm{AU}$ only. For the latter molecule, the column density is affected throughout the radial extent of the disk. For all molecules which possess an appreciable grainsurface column density, the values are relatively unaffected by the method used to compute the photochemical rates and the $\mathrm{X}$-ray ionization rate.

Overall, the recalculation of the photoreaction rates has a much bigger effect on the column densities than that of the $\mathrm{X}$-ray ionization rate with models $\mathrm{UV}$-old and $\mathrm{XR}+\mathrm{UV}$-old, on the whole, producing similar values and behavior. This is also true for models UV-new and $\mathrm{XR}+\mathrm{UV}$-new. Exceptions to this include $\mathrm{N}_{2} \mathrm{H}^{+}$(throughout the disk) and $\mathrm{OH}$ (in the outer disk beyond a radius of $\approx 50 \mathrm{AU})$. A further general observation is that the recalculation of the photorates affects each molecule in a different manner. The spectrum-dependent photorates do not only directly affect the abundance and distribution of atoms and molecules which can undergo photoionization and photodissociation, they also indirectly affect the subsequent gas-phase chemistry, leading to enhancements/depletions of molecules which are not directly formed or destroyed via a photochemical route, e.g., $\mathrm{HCO}^{+}$.

We see an interesting structure in the column densities of the sulfur-bearing species, CS and SO. Both show a peak between $\approx 2 \mathrm{AU}$ and $\sim 10 \mathrm{AU}$. In the outer disk, beyond $\sim 10 \mathrm{AU}$, sulfur exists primarily in the disk midplane as $\mathrm{H}_{2} \mathrm{~S}$ ice on the grain mantle. Within $10 \mathrm{AU}$, the disk midplane is warm enough for $\mathrm{H}_{2} \mathrm{~S}$ to evaporate from the grain mantle replenishing the gas with sulfur which forms $\mathrm{SO}$ and $\mathrm{CS}$. Within $\approx 2 \mathrm{AU}, \mathrm{SO}_{2}$ takes over from $\mathrm{SO}$ as the dominant gas-phase sulfur-bearing species. CS increases in abundance again within $\sim 1$ AU in the very warm, dense midplane.

The radial column densities, $N$, of a selection of molecules are listed in Table 4 at radii of $0.1,1,10$, and 100 AU. Molecules whose column densities are affected by more than one order of magnitude are highlighted in bold text. The general trend we see (with several exceptions discussed below) is a reduction in molecular column densities in the inner disk (within $1 \mathrm{AU}$ ) and an increase in the outer disk (beyond $1 \mathrm{AU}$ ) in models $\mathrm{UV}$-new and $\mathrm{XR}+\mathrm{UV}$-new relative to model UV-old. At $0.1 \mathrm{AU}$, $N\left(\mathrm{HCO}^{+}\right)$is reduced by two orders of magnitude with $N(\mathrm{OH})$ reduced by one order of magnitude, when the photochemistry is recalculated. The column density of $\mathrm{N}_{2} \mathrm{H}^{+}$is affected by both X-ray ionization and photochemistry with the former increasing the value relative to model UV-old and the latter reducing it. At $1 \mathrm{AU}$, only $N\left(\mathrm{~N}_{2} \mathrm{H}^{+}\right)$is significantly affected where it is enhanced by almost two orders of magnitude by the recalculation of the photochemistry and reduced by a factor of a few by the recalculation of the $\mathrm{X}$-ray ionization rate. At $10 \mathrm{AU}$, the molecules affected are $\mathrm{H}_{2} \mathrm{O}, \mathrm{CO}_{2}, \mathrm{CH}_{3} \mathrm{OH}$, and $\mathrm{N}_{2} \mathrm{H}^{+}$. The column density of $\mathrm{H}_{2} \mathrm{O}$ and $\mathrm{CO}_{2}$ are both enhanced by over an order of magnitude in models UV-new and $\mathrm{XR}+\mathrm{UV}$-new. As at $1 \mathrm{AU}, \mathrm{X}$-ray ionization reduces $N\left(\mathrm{~N}_{2} \mathrm{H}^{+}\right)$, whereas photochemistry enhances it. It is a similar story for methanol; however, X-ray ionization has a much smaller effect than photochemistry. At the final radius we consider here, $100 \mathrm{AU}$, again, $\mathrm{N}_{2} \mathrm{H}^{+}$and $\mathrm{CH}_{3} \mathrm{OH}$ are affected in a similar manner as at $10 \mathrm{AU}$. We also find that the column density of $\mathrm{OH}$ increased significantly in model UV-new with that for $\mathrm{CO}_{2}$ also enhanced in models UV-new and XR+UV-new. The only molecules for which $\mathrm{X}$-ray ionization significantly affects the column density are $\mathrm{N}_{2} \mathrm{H}^{+}$and $\mathrm{CH}_{3} \mathrm{OH}$.

The molecular column densities, although useful for tracing the general radial structure and identifying those molecules
Table 4

Column Densities

\begin{tabular}{|c|c|c|c|c|}
\hline Species & UV-old & XR+UV-old & UV-new & $\mathrm{XR+UV-new}$ \\
\hline \multicolumn{5}{|c|}{$0.1 \mathrm{AU}$} \\
\hline $\mathrm{H}$ & $1.4(21)$ & $1.4(21)$ & $4.5(20)$ & $4.2(20)$ \\
\hline $\mathrm{H}_{2}$ & $1.1(26)$ & $1.1(26)$ & $1.1(26)$ & $1.1(26)$ \\
\hline $\mathrm{CO}$ & $9.7(21)$ & $9.5(21)$ & $9.5(21)$ & $9.6(21)$ \\
\hline $\mathrm{HCO}^{+}$ & $2.1(14)$ & $2.5(14)$ & $3.2(12)$ & $1.7(12)$ \\
\hline $\mathrm{HCN}$ & $5.2(19)$ & $5.0(19)$ & $5.4(19)$ & $4.9(19)$ \\
\hline $\mathrm{CN}$ & $8.2(13)$ & $6.9(13)$ & $4.3(13)$ & $6.5(13)$ \\
\hline $\mathrm{CS}$ & $1.3(17)$ & $1.5(17)$ & $1.5(17)$ & $1.5(17)$ \\
\hline $\mathrm{C}_{2} \mathrm{H}$ & $1.0(15)$ & $9.9(14)$ & $9.6(14)$ & $9.8(14)$ \\
\hline $\mathrm{H}_{2} \mathrm{CO}$ & $6.9(15)$ & $8.2(15)$ & $6.5(15)$ & $8.3(15)$ \\
\hline $\mathbf{N}_{2} \mathbf{H}^{+}$ & 3.7(09) & $8.7(08)$ & $2.0(11)$ & $9.2(10)$ \\
\hline OH & $6.0(16)$ & $6.0(16)$ & $6.1(15)$ & $5.1(15)$ \\
\hline $\mathrm{H}_{2} \mathrm{O}$ & $2.8(22)$ & $2.8(22)$ & $2.8(22)$ & $2.8(22)$ \\
\hline $\mathrm{CO}_{2}$ & $3.8(18)$ & $5.4(18)$ & $3.7(18)$ & $5.5(18)$ \\
\hline $\mathrm{C}_{2} \mathrm{H}_{2}$ & $6.1(18)$ & $9.6(18)$ & $9.3(18)$ & $9.1(18)$ \\
\hline $\mathrm{CH}_{3} \mathrm{OH}$ & $2.3(18)$ & $2.4(18)$ & $2.3(18)$ & $2.4(18)$ \\
\hline \multicolumn{5}{|c|}{$1 \mathrm{AU}$} \\
\hline $\mathrm{H}$ & $1.1(21)$ & $1.1(21)$ & $8.8(20)$ & $8.3(20)$ \\
\hline $\mathrm{H}_{2}$ & $1.9(25)$ & $1.9(25)$ & $1.9(25)$ & $1.9(25)$ \\
\hline $\mathrm{CO}$ & $1.9(21)$ & $1.9(21)$ & $1.9(21)$ & $1.9(21)$ \\
\hline $\mathrm{HCO}^{+}$ & $1.7(14)$ & $1.9(14)$ & $9.4(13)$ & $6.5(13)$ \\
\hline $\mathrm{HCN}$ & $2.0(18)$ & $2.0(18)$ & $2.0(18)$ & $2.0(18)$ \\
\hline $\mathrm{CN}$ & $2.7(14)$ & $2.7(14)$ & $1.0(14)$ & $1.3(14)$ \\
\hline $\mathrm{CS}$ & $8.7(12)$ & $8.4(12)$ & $6.2(12)$ & $6.3(12)$ \\
\hline $\mathrm{C}_{2} \mathrm{H}$ & $4.1(14)$ & $4.4(14)$ & $9.1(13)$ & $1.5(14)$ \\
\hline $\mathrm{H}_{2} \mathrm{CO}$ & $3.1(13)$ & $3.1(13)$ & $3.2(13)$ & $3.1(13)$ \\
\hline $\mathbf{N}_{2} \mathbf{H}^{+}$ & $5.2(10)$ & 9.3(09) & $2.7(12)$ & $5.1(11)$ \\
\hline $\mathrm{OH}$ & $1.7(16)$ & $1.8(16)$ & $2.4(16)$ & $2.2(16)$ \\
\hline $\mathrm{H}_{2} \mathrm{O}$ & $2.2(21)$ & $2.2(21)$ & $2.2(21)$ & $2.2(21)$ \\
\hline $\mathrm{CO}_{2}$ & $7.7(20)$ & $7.7(20)$ & $7.7(20)$ & $7.7(20)$ \\
\hline $\mathrm{C}_{2} \mathrm{H}_{2}$ & $1.6(14)$ & $1.9(14)$ & $1.5(14)$ & $1.8(14)$ \\
\hline $\mathrm{CH}_{3} \mathrm{OH}$ & $1.0(15)$ & $1.0(15)$ & $1.0(15)$ & $1.0(15)$ \\
\hline \multicolumn{5}{|c|}{$10 \mathrm{AU}$} \\
\hline $\mathrm{H}$ & $7.3(20$ & $7.2(20)$ & $6.1(20)$ & $5.7(20)$ \\
\hline $\mathrm{H}_{2}$ & $2.6(24)$ & $2.6(24)$ & $2.6(24)$ & $2.6(24)$ \\
\hline $\mathrm{CO}$ & $2.0(20)$ & $2.0(20)$ & $2.0(20)$ & $2.0(20)$ \\
\hline $\mathrm{HCO}^{+}$ & $4.8(13)$ & $4.5(13)$ & $1.4(14)$ & $1.6(14)$ \\
\hline $\mathrm{HCN}$ & $8.0(14)$ & $2.1(15)$ & $4.5(14)$ & $6.9(14)$ \\
\hline $\mathrm{CN}$ & $7.3(13)$ & $8.1(13)$ & $3.0(14)$ & $3.4(14)$ \\
\hline $\mathrm{CS}$ & $4.7(13)$ & $4.4(13)$ & $4.3(13)$ & $5.5(13)$ \\
\hline $\mathrm{C}_{2} \mathrm{H}$ & $5.4(13)$ & $6.7(13)$ & 1.1(14) & $1.6(14)$ \\
\hline $\mathrm{H}_{2} \mathrm{CO}$ & $2.0(12)$ & $2.0(12)$ & $1.2(12)$ & $8.1(11)$ \\
\hline $\mathbf{N}_{2} \mathbf{H}^{+}$ & $2.0(10)$ & $5.7(09)$ & $2.8(11)$ & $9.2(10)$ \\
\hline $\mathrm{OH}$ & $8.9(15)$ & $9.4(15)$ & 2.1(16) & $2.3(16)$ \\
\hline $\mathbf{H}_{2} \mathbf{O}$ & $5.4(15)$ & $5.9(15)$ & $8.5(16)$ & $9.2(16)$ \\
\hline $\mathrm{CO}_{2}$ & $2.8(16)$ & $3.7(16)$ & $2.7(17)$ & $6.5(17)$ \\
\hline $\mathrm{C}_{2} \mathrm{H}_{2}$ & $7.5(13)$ & $3.2(13)$ & $7.9(13)$ & $3.7(13)$ \\
\hline $\mathrm{CH}_{3} \mathrm{OH}$ & $1.0(08)$ & $9.1(07)$ & $5.3(09)$ & $5.2(09)$ \\
\hline \multicolumn{5}{|c|}{$100 \mathrm{AU}$} \\
\hline $\mathrm{H}$ & $3.3(19)$ & 1.7(19) & $3.2(19)$ & $1.5(19)$ \\
\hline $\mathrm{H}_{2}$ & $2.0(23)$ & $2.0(23)$ & $2.0(23)$ & $2.0(23)$ \\
\hline $\mathrm{CO}$ & $9.1(18)$ & $9.7(18)$ & $8.9(18)$ & $9.3(18)$ \\
\hline $\mathrm{HCO}^{+}$ & $3.9(13)$ & $3.6(13)$ & $5.8(13)$ & $4.3(13)$ \\
\hline $\mathrm{HCN}$ & $4.8(14)$ & $2.5(14)$ & $2.3(14)$ & 2.1(14) \\
\hline $\mathrm{CN}$ & $2.4(14)$ & $2.6(14)$ & $5.0(14)$ & $4.1(14)$ \\
\hline $\mathrm{CS}$ & $3.1(13)$ & $3.7(13)$ & $1.8(13)$ & $1.7(13)$ \\
\hline $\mathrm{C}_{2} \mathrm{H}$ & $1.0(14)$ & $1.2(14)$ & 7.7(13) & $5.4(13)$ \\
\hline $\mathrm{H}_{2} \mathrm{CO}$ & $4.4(12)$ & $3.7(12)$ & $8.2(12)$ & $2.9(12)$ \\
\hline $\mathbf{N}_{2} \mathbf{H}^{+}$ & $1.9(11)$ & $8.8(10)$ & $1.5(12)$ & $5.5(11)$ \\
\hline OH & $9.0(14)$ & $3.0(14)$ & $6.3(15)$ & $3.3(15)$ \\
\hline $\mathrm{H}_{2} \mathrm{O}$ & $4.9(16)$ & $2.5(16)$ & $3.1(16)$ & $1.4(16)$ \\
\hline $\mathrm{CO}_{2}$ & $2.2(15)$ & $2.1(15)$ & $5.9(16)$ & $8.8(16)$ \\
\hline $\mathrm{C}_{2} \mathrm{H}_{2}$ & $3.0(13)$ & $3.1(13)$ & $2.4(13)$ & $1.6(13)$ \\
\hline $\mathrm{CH}_{3} \mathrm{OH}$ & 4.1(10) & $4.0(11)$ & $7.9(10)$ & 2.1(11) \\
\hline
\end{tabular}

Note. $a(b)$ means $a \times 10^{b}$. 
significantly affected by the inclusion or omission of each process, provide little information on the spatial distribution and abundance. This ultimately affects the strength of the line emission from the disk since this is influenced by the physical conditions in the region where each molecule is most abundant. In the following section, we look more closely at the effects on the two-dimensional molecular structure of the disk due to the recalculation of the photoreaction and X-ray ionization rates.

\subsection{Photochemistry}

In Figure 4 we display the fractional abundance of $\mathrm{HCO}^{+}$, $\mathrm{OH}, \mathrm{H}_{2} \mathrm{O}, \mathrm{CO}_{2}$, and $\mathrm{N}_{2} \mathrm{H}^{+}$as a function of disk radius and height (scaled by the radius) for models UV-old (left column) and UV-new (right column). These molecules are those we have identified as being most affected by the recalculation of the photorates.

In the plots for $\mathrm{HCO}^{+}$, it is clearly seen that within a radius of $\approx 1 \mathrm{AU}$, the fractional abundance of $\mathrm{HCO}^{+}$in the "molecular layer" in this region, located at $Z / R=0.1$, is around two orders of magnitude lower in model UV-new than in model UV-old. Also clearly visible is the reason for the larger column density calculated beyond this radius, the depth of the layer of $\mathrm{HCO}^{+}$ in model UV-new is much larger than that in model UV-old although the maximum fractional abundance attained in both models is similar $\left(x\left(\mathrm{HCO}^{+}\right) \sim 10^{-6}\right)$. The abundance of $\mathrm{HCO}^{+}$ is controlled by ion-molecule chemistry and thus depends on the abundance of ionic and neutral precursors. An example of an ion-molecule gas-phase reaction which leads to the production of $\mathrm{HCO}^{+}$is

$$
\mathrm{CO}+\mathrm{H}_{3}^{+} \longrightarrow \mathrm{HCO}^{+}+\mathrm{H}_{2}
$$

In model UV-new, where we see an increase in $\mathrm{HCO}^{+}$, we see a corresponding increase in $\mathrm{CO}$ and $\mathrm{H}_{3}^{+}$. $\mathrm{CO}$ is directly influenced by the photochemistry since it can be photodissociated to produce $\mathrm{C}$ and $\mathrm{O} . \mathrm{H}_{3}^{+}$, on the other hand, is primarily formed via the reaction of $\mathrm{H}_{2}$ with $\mathrm{H}_{2}^{+}$and destroyed via electron recombination. The enhancement in $\mathrm{HCO}^{+}$in model $\mathrm{UV}$-new also corresponds to where we see a slight decrease in electron abundance (see Figure 7). Relating this back to the photochemistry, this indicates an overestimation in this region in both the photodissociation of $\mathrm{CO}$ and photoionization (in general) in our fiducial model (model UV-old).

The depth of the molecular layer where $\mathrm{OH}$ reaches its maximum fractional abundance $\left(x(\mathrm{OH}) \sim 10^{-4}\right)$ within $1 \mathrm{AU}$ is smaller in model UV-old than in model UV-new, whereas, beyond this radius, the depth is larger. This accounts for the smaller column density in model UV-new at $1 \mathrm{AU}$ and the larger at 10 and $100 \mathrm{AU}$. The distribution of $\mathrm{OH}$ is also somewhat different in model UV-new, reaching an order-of-magnitudehigher fractional abundance in the disk surface throughout the radial extent. The abundance of $\mathrm{OH}$ is directly controlled by the photochemistry since $\mathrm{OH}$ is one of the products of the photodissociation of $\mathrm{H}_{2} \mathrm{O}$ and can itself be photodissociated to form $\mathrm{O}$ and $\mathrm{H}$. We see in the corresponding plots for water in Figure 4 that its fractional abundance is also slightly higher in the disk surface in model UV-new relative to model UV-old. We also find that the abundance of free oxygen atoms decreases in this region, indicating in model UV-new, more atomic oxygen is locked up in oxygen-bearing molecules than in model UV-old.

Looking at the distribution of water, we see a large enhancement in the fractional abundance in the molecular layer beyond a radius of $\approx 1 \mathrm{AU}$, going from a value of $\sim 10^{-6}$ in model UV-old to $\sim 10^{-4}$ in model UV-new. Not only is the maximum fractional abundance reached much higher, the extent over which water exists with a value $\gtrsim 10^{-9}$ in model UV-new is also much larger. We conclude that the abundance of gas-phase water is very sensitive to the method employed to calculate the photorates.

Both $\mathrm{OH}$ and $\mathrm{H}_{2} \mathrm{O}$ can be formed via neutral-neutral gasphase reactions in warm regions of the disk where $T \gtrsim 200 \mathrm{~K}$ (Glassgold et al. 2009):

$$
\begin{gathered}
\mathrm{H}_{2}+\mathrm{O} \longrightarrow \mathrm{OH}+\mathrm{H} \\
\mathrm{H}_{2}+\mathrm{OH} \longrightarrow \mathrm{H}_{2} \mathrm{O}+\mathrm{H} .
\end{gathered}
$$

Since we see an increase in the fractional abundance of $\mathrm{H}_{2}$ in model UV-new relative to UV-old over the region where both $\mathrm{OH}$ and $\mathrm{H}_{2} \mathrm{O}$ are increased, this gas-phase production route for both species is more important in model UV-new than in model $\mathrm{UV}$-old. Hence, the enhancement seen in the abundances of $\mathrm{OH}$ and $\mathrm{H}_{2} \mathrm{O}$ in model UV-new is a combination of increased gasphase production and decreased photodestruction. In Figure 5, we display the photodissociation rates of $\mathrm{OH}$ and $\mathrm{H}_{2} \mathrm{O}$ as a function of disk height (scaled by the radius) at radii of $1 \mathrm{AU}$, $10 \mathrm{AU}$, and $100 \mathrm{AU}$. A decrease of around an order of magnitude in the photodissociation rates of both species is clearly seen in the upper disk layers accounting for the increase in abundance of both species in this region. The gross overestimation of the photodissociation rates in model UV-old is due to the inclusion of Ly $\alpha$ photons in the calculation of the wavelengthintegrated UV photon flux (see Section 2.2.1). Also, note that the dissociation rates vary differently as a function of height in model UV-new versus UV-old since in the former model, the wavelength dependence of both the photo cross sections and UV field are included.

Gas-phase $\mathrm{CO}_{2}$ is affected mainly in the outer disk beyond a radius of $\approx 10 \mathrm{AU}$. We see that $\mathrm{CO}_{2}$ in this region exists in a layer lower than that of water (due to its lower binding energy to dust grains) and is enhanced in the outer disk from a fractional abundance $\sim 10^{-6}$ in model UV-old to $\sim 10^{-4}$ in model UV-new. In model UV-new, in this region, it possesses a comparable fractional abundance to the other main oxygen-bearing species, $\mathrm{CO}$ and $\mathrm{O}_{2}$. As in the case for water, the extent over which $x\left(\mathrm{CO}_{2}\right)$ has a value $\gtrsim 10^{-9}$ is much larger in model UV-new than in model UV-old. We see an enhancement in $\mathrm{CO}_{2}$ when the fractional abundance of ionic carbon begins to increase relative to atomic carbon. Note that $\mathrm{CO}_{2}$ is destroyed via reaction with $\mathrm{C}^{+}$to form $\mathrm{CO}^{+}$and $\mathrm{CO}$. In model UV-new, the boundary where $\mathrm{C}^{+} / \mathrm{C} \sim 1$ is much higher in the disk than in model UV-old (at a height of $\approx 5 \mathrm{AU}$ and $\approx 2 \mathrm{AU}$, respectively, at a radius of $10 \mathrm{AU}$ ). Thus in model UV-new, gas-phase $\mathrm{CO}_{2}$ can evaporate from the grain surface and remain intact in this layer due to the lack of sufficient gas-phase destruction mechanisms. We display the photodissociation rate of $\mathrm{CO}_{2}$ as a function of disk height (scaled by the radius) at radii of $1 \mathrm{AU}, 10 \mathrm{AU}$, and $100 \mathrm{AU}$ in Figure 5. As in the case for $\mathrm{OH}$ and $\mathrm{H}_{2} \mathrm{O}$, we see a decrease of around an order of magnitude in model UV-new compared with UV-old. This accounts for the increase in the abundance of $\mathrm{CO}_{2}$ in the upper disk layers in model UV-new. Note that in model UV-old, the dissociation rates for $\mathrm{CO}_{2}$ are consistently larger than that of $\mathrm{H}_{2} \mathrm{O}$ and $\mathrm{OH}$, reflecting the rates calculated for the unshielded interstellar medium $\left(14,5.9\right.$, and $3.5 \times 10^{-10} \mathrm{~s}^{-1}$, respectively). However, in model UV-new, the $\mathrm{H}_{2} \mathrm{O}$ photodissociation has the largest rate at each radius, with $\mathrm{OH}$ also having a larger rate than $\mathrm{CO}_{2}$ at $100 \mathrm{AU}$. This demonstrates how the relative photorates are sensitive to the shape of the radiation field due to the specific variation in the photo cross section of each species. 

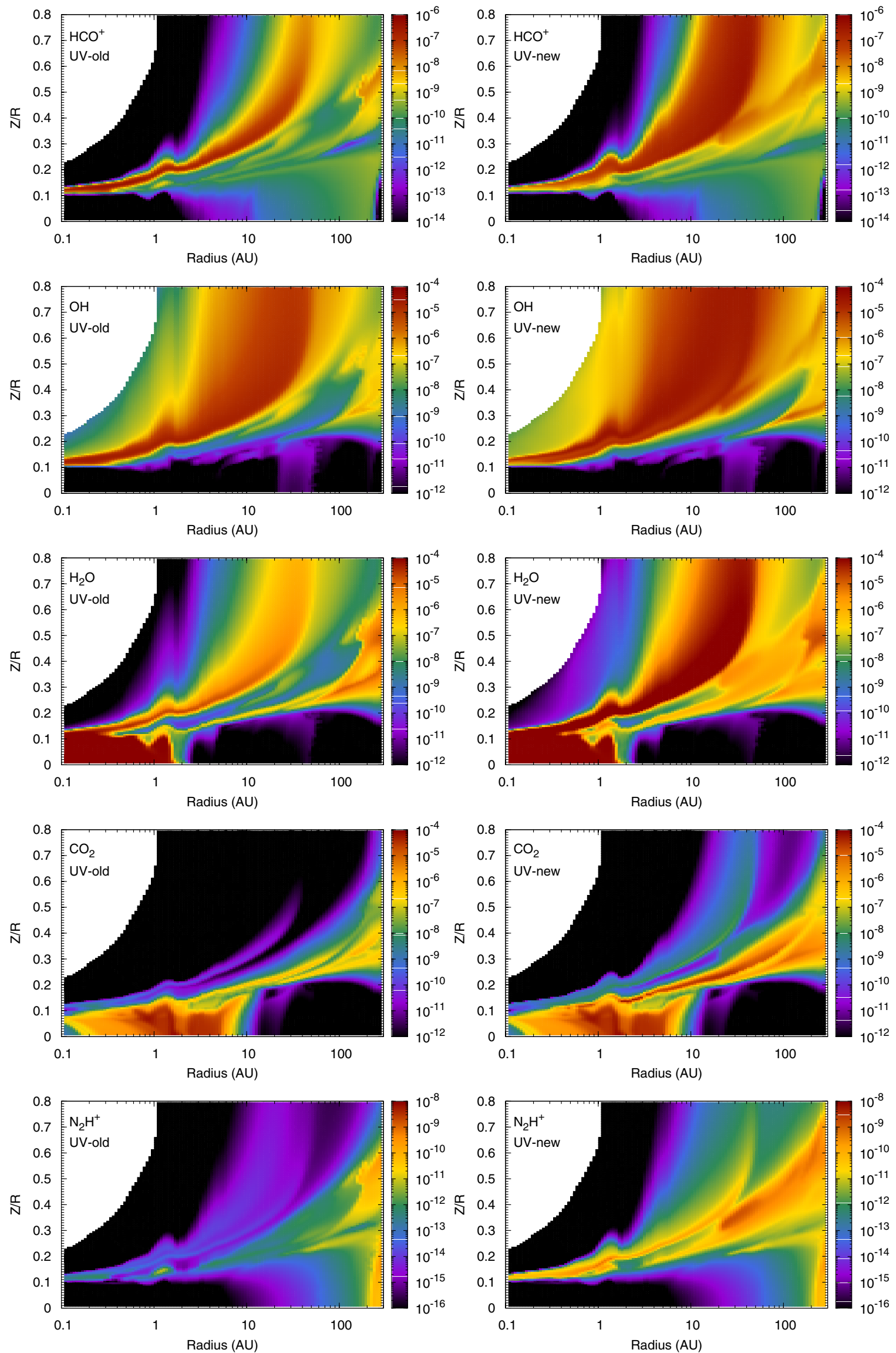

Figure 4. Fractional abundance of $\mathrm{HCO}^{+}, \mathrm{OH}, \mathrm{H}_{2} \mathrm{O}, \mathrm{CO}_{2}$, and $\mathrm{N}_{2} \mathrm{H}^{+}$as a function of disk radius, $R$, and height (scaled by the radius, i.e., $Z / R$ ) for models $\mathrm{UV}$-old (left) and UV-new (right).

(A color version of this figure is available in the online journal.) 


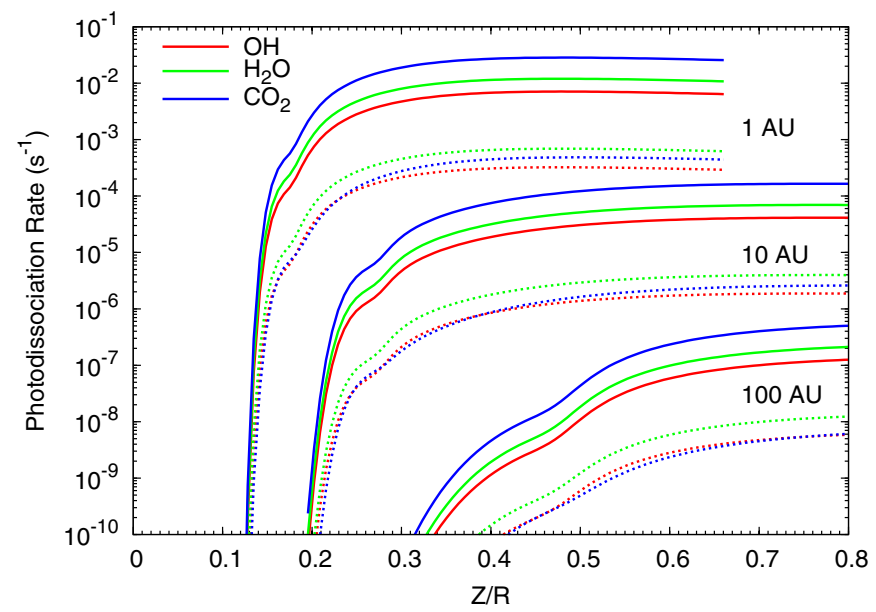

Figure 5. Photodissociation rates of $\mathrm{OH}, \mathrm{H}_{2} \mathrm{O}$, and $\mathrm{CO}_{2}$ as a function of disk height (scaled by the radius, i.e., $Z / R$ ) at radii of $1 \mathrm{AU}, 10 \mathrm{AU}$, and $100 \mathrm{AU}$ for models UV-old (solid lines) and UV-new (dashed lines).

(A color version of this figure is available in the online journal.)

When the photochemistry is recalculated, we see a dramatic increase in the region over which $\mathrm{N}_{2} \mathrm{H}^{+}$reaches its maximum fractional abundance, now residing in a molecular layer which permeates the entire disk. The most dramatic increase is seen in the inner disk $(<100 \mathrm{AU})$ where $x\left(\mathrm{~N}_{2} \mathrm{H}^{+}\right)$jumps from a maximum value of $\sim 10^{-12}$ in model UV-old to $\sim 10^{-9}$ in model UV-new. Similar to $\mathrm{HCO}^{+}$, the abundance of $\mathrm{N}_{2} \mathrm{H}^{+}$is controlled by ion-molecule chemistry, it is formed via the reaction of $\mathrm{N}_{2}$ with $\mathrm{H}_{3}^{+}$, and it is destroyed effectively by reaction with $\mathrm{CO}$ and electron recombination. Looking at Figure 7 which displays the electron fractional abundance for all four models as a function of disk radius and height, we see in the layer where $\mathrm{N}_{2} \mathrm{H}^{+}$is significantly enhanced, there is a respective decrease in the electron fraction in model $\mathrm{UV}$-new. $\mathrm{N}_{2} \mathrm{H}^{+}$and $\mathrm{HCO}^{+}$ show different behaviors since the abundance of $\mathrm{N}_{2} \mathrm{H}^{+}$is more sensitive to the abundance of $\mathrm{H}_{3}^{+}$than $\mathrm{HCO}^{+}$. $\mathrm{HCO}^{+}$has various alternative routes to formation other than the reaction of $\mathrm{CO}$ and $\mathrm{H}_{3}^{+}$, e.g., $\mathrm{C}^{+}+\mathrm{H}_{2} \mathrm{O}$, whereas $\mathrm{N}_{2} \mathrm{H}^{+}$forms mainly via the reaction of $\mathrm{N}_{2}$ with $\mathrm{H}_{3}^{+}$. We discuss $\mathrm{N}_{2} \mathrm{H}^{+}$chemistry further in Section 3.3.

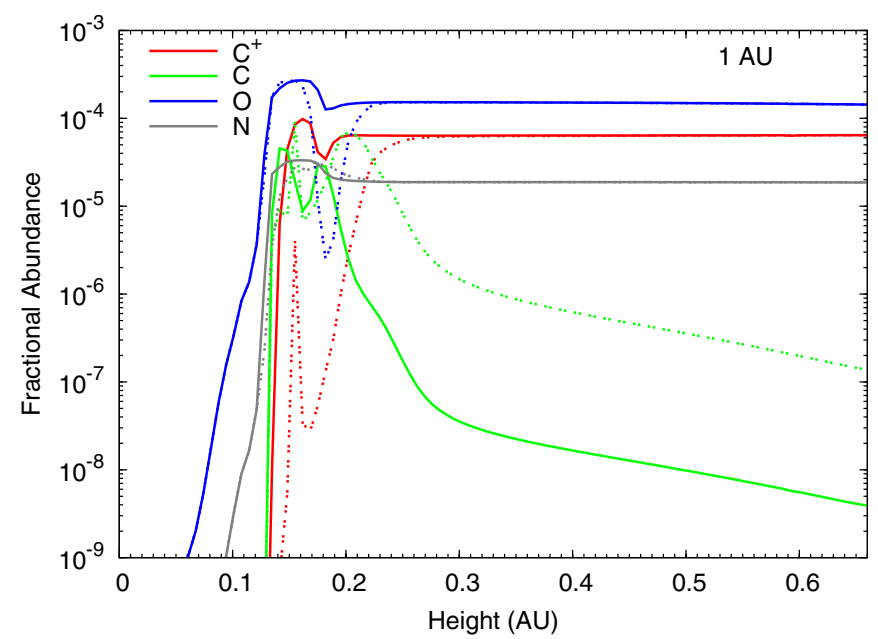

In Figure 6, we present the fractional abundances of several atoms and ions at a radius of 1 (top panel) and $10 \mathrm{AU}$ (bottom panel). We display the results for $\mathrm{C}^{+}$also since the ionization potential for carbon is much lower than that for oxygen and nitrogen. In model UV-new, there is a significant depletion of free $\mathrm{O}$ atoms and $\mathrm{C}^{+}$ions in the region where we see an increase in the fractional abundance of $\mathrm{OH}, \mathrm{H}_{2} \mathrm{O}$, and the main carbon species, $\mathrm{C}$. Note that the atomic carbon abundance in the disk surface is larger in model UV-new than in model UV-old; however, here free carbon exists mainly in ionic form as $\mathrm{C}^{+}$. In this region, we also see an enhancement in the fractional abundances of the molecular ions, $\mathrm{HCO}^{+}$and $\mathrm{N}_{2} \mathrm{H}^{+}$. Hence, in model UV-new, in the molecular layer where molecules reach their maximum fractional abundance, more oxygen atoms are contained within molecules than in model UV-old. Also, in this region, less carbon exists as $\mathrm{C}^{+}$so the ratio of $\mathrm{C}^{+} / \mathrm{C}$ is generally lower in model UV-new than in model UV-old. This can be attributed to (1) the photorates in model UV-old being overestimated for particular molecules and atoms and (2) an adjustment of the gas-phase chemistry due to the enhancement/depletion of species directly affected by the radiation field.

The layer in which there is an enhancement in the abundance of molecules also coincides with the boundary where we cross from hydrogen in mainly molecular form $\left(\mathrm{as} \mathrm{H}_{2}\right)$ to atomic form. In model UV-new, this transition is smoother and results in a slightly lower fractional abundance of $\mathrm{H}$ atoms over the extent of the molecular layer. At high temperatures, the destruction of molecules by free hydrogen atoms can become an important process. The region where we find the large increase in $\mathrm{OH}$ and $\mathrm{H}_{2} \mathrm{O}$ coincides with a gas temperature of $\gtrsim 1000 \mathrm{~K}$ so that the reduction of free $\mathrm{H}$ atoms (which act to destroy molecules) in model UV-new is also influencing the gas-phase chemistry and subsequent abundances.

\subsection{X-Ray Ionization}

We find that the only molecule significantly affected by X-ray ionization is $\mathrm{N}_{2} \mathrm{H}^{+}$. We see a decrease in both the maximum fractional abundance attained by $\mathrm{N}_{2} \mathrm{H}^{+}$and a reduction in the spatial extent over which it exists with an appreciable abundance. Note that in model UV-old, the distribution of $\mathrm{N}_{2} \mathrm{H}^{+}$ is also different to that of any of the other molecules discussed

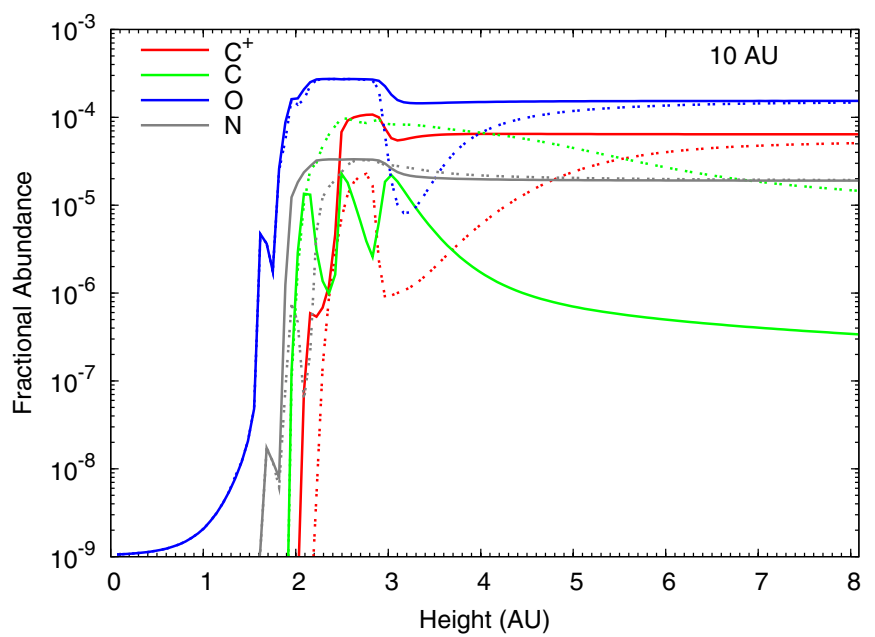

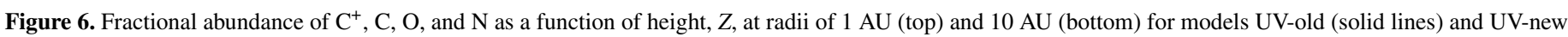
(dashed lines).

(A color version of this figure is available in the online journal.) 

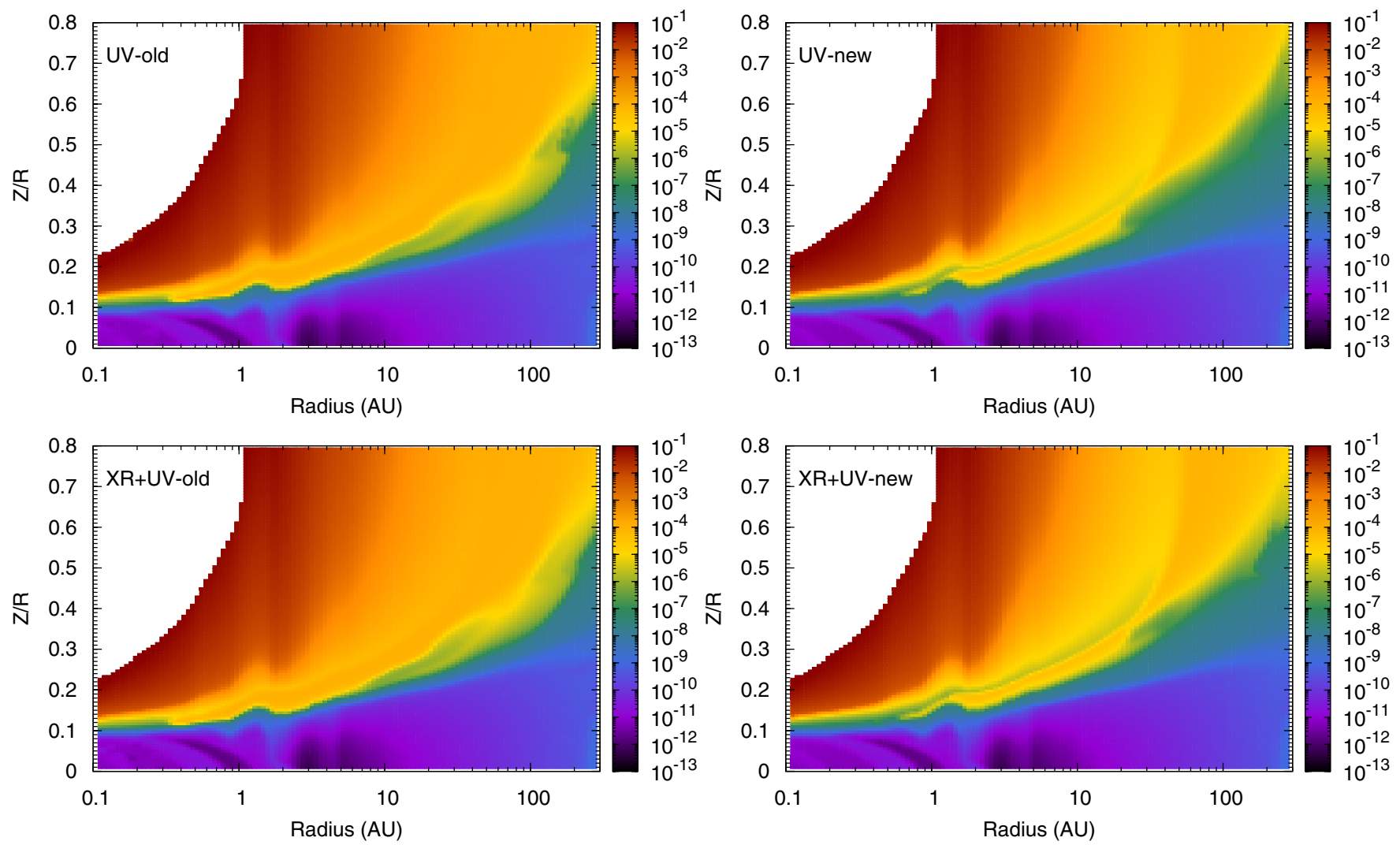

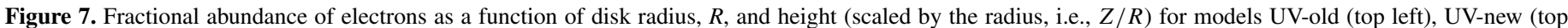
right), $\mathrm{XR}+\mathrm{UV}$-old (bottom left), and $\mathrm{XR}+\mathrm{UV}$-new (bottom right).

(A color version of this figure is available in the online journal.)

thus far, existing mainly in the outer disk beyond a radius of $\approx 100 \mathrm{AU}$ and extending into the disk midplane (see Figure 4). Since the abundance of $\mathrm{N}_{2} \mathrm{H}^{+}$is controlled by ion-molecule chemistry, the relative abundances of precursor ions and neutral molecules will influence the amount of $\mathrm{N}_{2} \mathrm{H}^{+}$which can exist. $\mathrm{N}_{2} \mathrm{H}^{+}$is formed in cold, dense regions via reaction of $\mathrm{H}_{3}^{+}$with $\mathrm{N}_{2}$, the latter of which is formed via radical-radical reactions, e.g., $\mathrm{N}+\mathrm{NH} \rightarrow \mathrm{N}_{2}+\mathrm{H}$. The abundance of $\mathrm{H}_{3}^{+}$is controlled by the ionization of $\mathrm{H}_{2}$ to form $\mathrm{H}_{2}^{+}$. In reactions where molecular ions can form via proton donation, the ionization rate can have a large impact on the resulting abundances reached, as seen here for the case of $\mathrm{N}_{2} \mathrm{H}^{+}$. Also, $\mathrm{N}_{2} \mathrm{H}^{+}$is effectively destroyed by electron recombination so that the ionization fraction in the disk plays an important role (see Section 3.4). However, we do not see this effect in the abundance and distribution of $\mathrm{HCO}^{+}$, which also depends, albeit to a lesser extent (see below), on the abundance of $\mathrm{H}_{3}^{+}$and which appears independent of the treatment of $\mathrm{X}$-ray ionization. $\mathrm{HCO}^{+}$resides in a different layer in the disk where the abundance and distribution of $\mathrm{H}_{3}^{+}$and electrons do not vary significantly between models $\mathrm{UV}$-old and $\mathrm{XR}+\mathrm{UV}$-old. We do see corresponding differences in the abundance and distribution of $\mathrm{H}_{3}^{+}$, in particular, between models $\mathrm{UV}$-old and $\mathrm{XR}+\mathrm{UV}$-old in the region where $\mathrm{N}_{2} \mathrm{H}^{+}$is most abundant. $\mathrm{HCO}^{+}$ also has a plethora of different routes to formation, other than the protonation of $\mathrm{CO}$ by $\mathrm{H}_{3}^{+}$.

In our disk model, $\mathrm{X}$-ray ionization rate dominates in the outer disk upper layers over cosmic-ray ionization. Our recalculation of the rates in this region leads to a decrease in $\mathrm{N}_{2} \mathrm{H}^{+}$indicating an overestimation of the $\mathrm{X}$-ray ionization rate in our original model.

\subsection{Disk Ionization Fraction}

In Figure 7 we display the fractional abundance of electrons as a function of disk radius and height for all models considered in this work and in Figure 8, we present the fractional abundances of several abundant cations as a function of disk height at radii of $15 \mathrm{AU}$ (top) and $150 \mathrm{AU}$ (bottom).

At first glance, there appears to be little difference in the distribution of electrons between the different models. The ionization fraction in the disk ranges from close to 1 , in the disk surface nearest the star, to less than $10^{-12}$ in the disk midplane between a radius of $1 \mathrm{AU}$ and $10 \mathrm{AU}$. Comparing the plots for models UV-old and XR+UV-old, we see only minor differences; in the boundary layer where the ionization gradient is highest and coinciding with the aforementioned molecular layer, and in the midplane layer where the electron fraction reaches values $<10^{-11}$, which is slightly larger in depth in model XR+UV-old.

Comparing models UV-old and UV-new we see that there is more of an effect in the boundary layer described above, which extends into the outer disk; in fact, we see that at the outermost radius, $\approx 300 \mathrm{AU}$, the ionization fraction in the disk surface layer $(Z / R \approx 0.6-0.7)$ in model UV-new is orders of magnitude lower than that in model UV-old. This is coincident with where we see an increase in the abundance of $\mathrm{N}_{2} \mathrm{H}^{+}$. We also note the slight increase in the electron abundance in the disk surface in model UV-new compared with model UV-old. This is unsurprising, as we would expect the stellar radiation field to impact on the chemistry in the disk surface, in particular. Comparing the graphs for models UV-new and XR+UV-new, we see the effects of the X-ray ionization in the disk surface, 

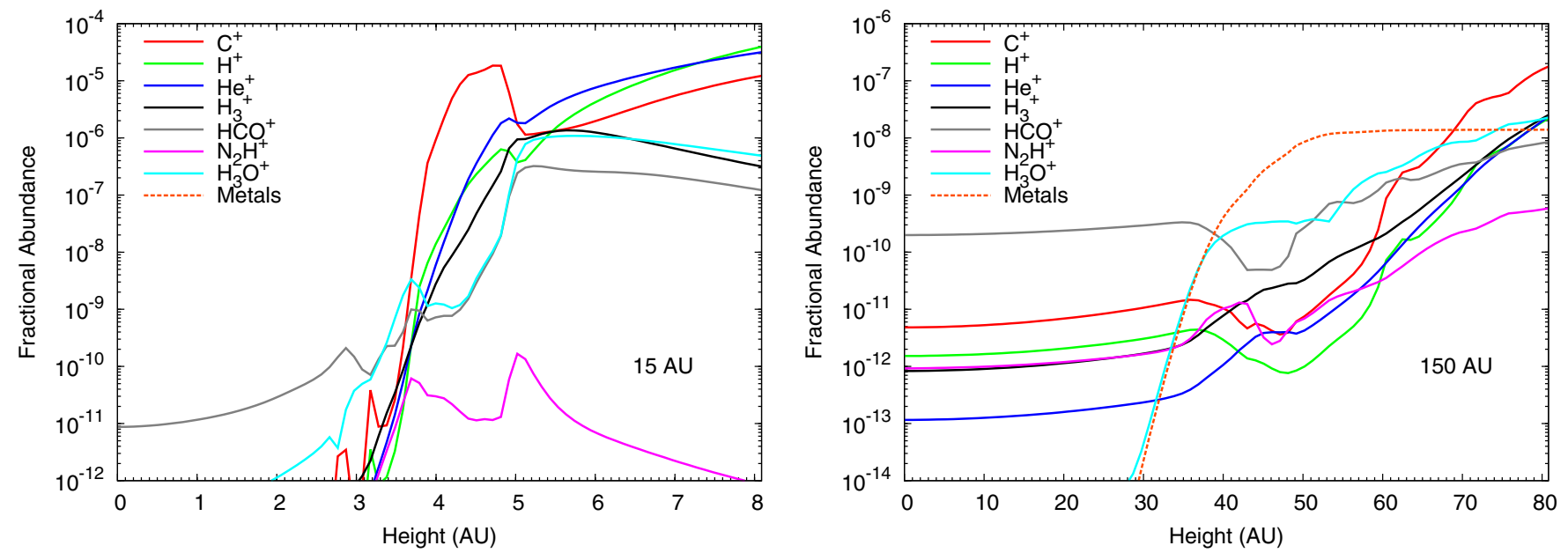

Figure 8. Fractional abundance of several important cations as a function of height, $Z$, at radii of 15 AU (top) and 150 AU (bottom) for model UV-new.

(A color version of this figure is available in the online journal.)
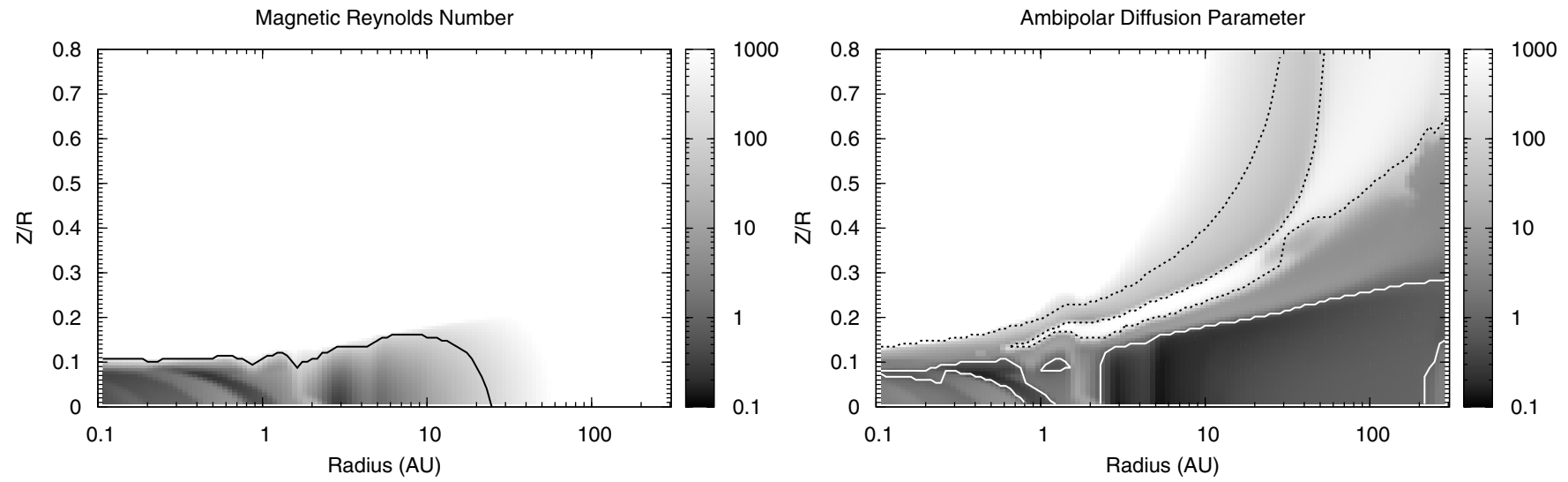

Figure 9. Magnetic Reynolds number, $\mathrm{Re}_{\mathrm{M}}$ (left), and ambipolar diffusion parameter, Am (right), as a function of disk radius, $R$, and height (scaled by the radius, i.e., $Z / R)$ for model XR+UV-new. The black contours on both plots represent the boundary where $\mathrm{Re}_{\mathrm{M}}$ and $\mathrm{Am}=100$, whereas the white contours on the right-hand plot represent the boundary where $\mathrm{Am}=1$.

which negates the decrease in the electron abundance to some extent.

Looking at Figure 8, in the disk surface at $15 \mathrm{AU}$, the most abundant cations are $\mathrm{H}^{+}$and $\mathrm{He}^{+}$whereas at $150 \mathrm{AU}$, the most abundant is $\mathrm{C}^{+}$. In the disk midplane, at all radii $\gtrsim 1 \mathrm{AU}, \mathrm{HCO}^{+}$ dominates the cation fraction. Within $\sim 1 \mathrm{AU}$, metallic ions such as $\mathrm{Fe}^{+}$and $\mathrm{Na}^{+}$also contribute to the cationic abundance in the midplane forming efficiently via charge exchange (at the expense of molecular cations such as $\mathrm{HCO}^{+}$). In the molecular layer, the picture is more complex with several molecular and atomic ions contributing to the cation fraction including $\mathrm{H}_{3}^{+}$, $\mathrm{H}_{3} \mathrm{O}^{+}, \mathrm{HCO}^{+}$, and $\mathrm{C}^{+}$. In this region, at $150 \mathrm{AU}$, metallic ions with an appreciable fractional abundance $\left(\sim 10^{-9}\right.$ to $\left.10^{-8}\right)$ such as $\mathrm{Fe}^{+}, \mathrm{Mg}^{+}$, and $\mathrm{Na}^{+}$also contribute to the cation fraction. $\mathrm{N}_{2} \mathrm{H}^{+}$ only reaches significant abundances in the outer disk ( $\gtrsim 100 \mathrm{AU})$ caused, to a degree, by the depletion of gas-phase CO due to freezeout onto grain surfaces in this region.

Following the theory outlined in Section 2.3, we calculated the magnetic Reynolds number, $\mathrm{Re}_{\mathrm{M}}$, and the ambipolar diffusion parameter, Am, as a function of disk radius and height and our results are displayed in Figure 9. We plot the results for model $\mathrm{XR}+\mathrm{UV}$-new only, since we find that both the photochemistry and X-ray ionization have little impact on the values of $\mathrm{Re}_{\mathrm{M}}$ and Am in the inner disk midplane.

Simulations by Sano \& Stone (2002) suggest that a suitable value for $\operatorname{Re}_{\mathrm{M}}^{\text {crit }}$ is $\approx 100$. At values lower than this, accretion in the disk is likely inhibited due to suppression of the MRI by Ohmic dissipation. Looking at the left panel of Figure 9 and adopting this criterion, we see that there is a probable dead zone where the MRI is susceptible to Ohmic dissipation in the midplane extending from the innermost radius, $0.1 \mathrm{AU}$, to $\approx 20 \mathrm{AU}$. The depth of this dead zone varies from $Z / R=0.1$ at 0.1 AU to $Z / R \approx 0.15$ at $10 \mathrm{AU}$. We find little difference in the extent of the dead zone between the various chemical models.

Similarly, the simulations of Bai \& Stone (2011) suggest that ambipolar diffusion can inhibit mass and angular momentum transport in regions in the disk where the ambipolar diffusion parameter, Am, exceeds a critical value, $\mathrm{Am}^{\text {crit }} \sim 1$. In the right-hand panel of Figure 9, the white contours represent the boundary where $\mathrm{Am}=1$ whereas the black contours represent $\mathrm{Am}=100$. We see ambipolar diffusion has a bigger effect creating a much larger dead zone in the disk midplane, ranging from just beyond $2 \mathrm{AU}$ out to around $200 \mathrm{AU}$. If we adopt the earlier criterion of Hawley \& Stone (1998), i.e., $\mathrm{Am}^{\text {crit }}=100$ (black contours), we find that the MRI is suppressed throughout most of the disk mass.

Perez-Becker \& Chiang (2011) argue that, in order for efficient accretion in protoplanetary disks, both criteria for $\mathrm{Re}_{\mathrm{M}}$ and Am must be met. Our calculations suggest that in our disk model, most of the midplane is inactive and so accretion can occur via the surface layers only, a similar result to that found by Gammie (1996). As a measure of the effect on the mass accretion 


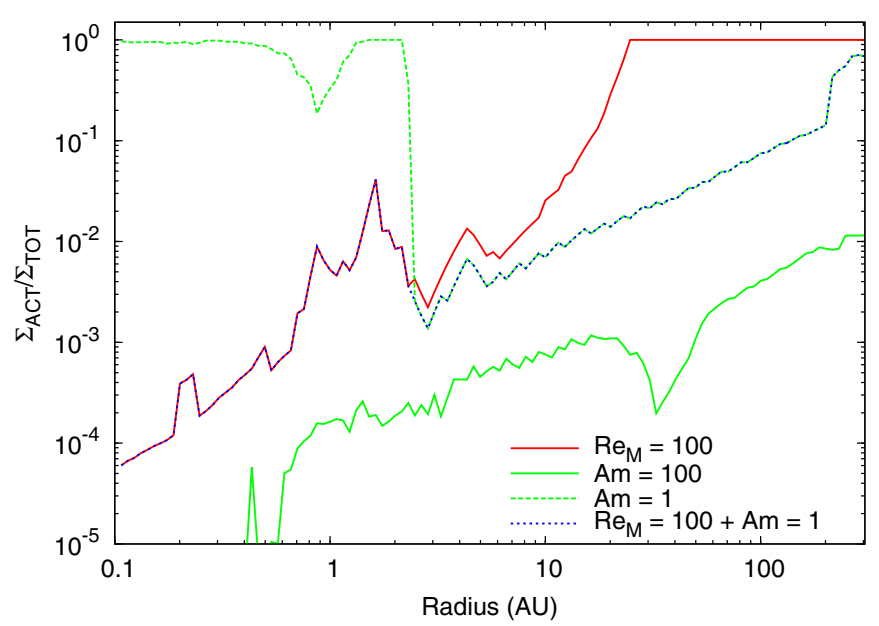

Figure 10. Ratio of the active column density to the total column density, $\Sigma_{\mathrm{ACT}} / \Sigma_{\mathrm{TOT}}$, as a function of radius, $R$, for four criterion: $\operatorname{Re}_{\mathrm{M}}>100$ (solid red line), Am > 100 (solid green line), Am $>1$ (dashed green line), and $\operatorname{Re}_{\mathrm{M}}>100$ and Am $>1$.

(A color version of this figure is available in the online journal.)

rate of the disk due to the presence of dead zones, in Figure 10 we show the ratio of the "active" column density to the total column density, $\Sigma_{\mathrm{ACT}} / \Sigma_{\mathrm{TOT}}$, as a function of disk radius, $R$, since this ratio estimates the efficiency of accretion. We plot $\Sigma_{\mathrm{ACT}}$ for four criteria: $\mathrm{Re}_{\mathrm{M}}>100$ (solid red line), $\mathrm{Am}>100$ (solid green line), $\mathrm{Am}>1$ (dashed green line), and $\mathrm{Re}_{\mathrm{M}}>100$ and $\mathrm{Am}>1$ (dotted blue line). For the latter criterion, we see that accretion efficiency ranges from $\lesssim 1 \%$ in the inner disk $(<10 \mathrm{AU})$ to around $70 \%$ in the outer disk ( $>200 \mathrm{AU})$. Although most of the outer disk is unaffected by Ohmic dissipation (solid red line), it is susceptible to ambipolar diffusion (dashed green line). Applying the more stringent condition of Am $>100$ (solid green line), we find that most of the disk is constrained to an accretion rate around $1 \%$ of the rate assumed in the calculation of the disk structure. The structure seen in the active column density is due to the corresponding structure seen in the spatial distribution of $\mathrm{Re}_{\mathrm{M}}$ and Am (displayed in Figure 9) which are related to the fractional and absolute electron density, respectively.

Compared with Ohmic dissipation, ambipolar diffusion suppresses the MRI in lower density regions (see, e.g., Figure 1 in Kunz \& Balbus 2004). Our results are consistent with this since we see the dead zone extending into the lower density regions in our disk, i.e., to larger radii in the disk midplane and toward the disk surface, when the ambipolar diffusion criterion is included.

The suppression of accretion in specific areas leading to a buildup of material flowing through the disk midplane will change the physical nature of the disk. This, in turn, influences the chemical composition and thus the penetration of UV photons, X-ray photons, and cosmic-ray particles. The changing physical and chemical conditions may act to increase $\mathrm{Re}_{\mathrm{M}}$ and Am to the extent that they exceed their critical values; hence, the presence of dead zones may be time dependent. Young stars and protostellar objects often undergo transient periods of enhancements in luminosity often ascribed to spells where the accretion disk possesses an increased mass flow rate going from $\lesssim 10^{-7} M_{\odot} \mathrm{yr}^{-1}$ to $\sim 10^{-4} M_{\odot} \mathrm{yr}^{-1}$ for periods of $\sim 100$ yr. An episodic buildup and release of material in the disk due to the development and subsequent dissipation of an accompanying dead zone may be responsible (see, e.g., Herbig 1977; Hartmann \& Kenyon 1996; Calvet et al. 2000; Armitage et al. 2001; Vorobyov \& Basu 2006).
We note here that the steady disk model is a first step in our attempt to approach reality. A more sophisticated model, in which we treat the charge balance more realistically (including detailed balance of grain charge and polycyclic aromatic hydrocarbons (PAHs)) and in which we calculate the dust evolution in conjunction with the chemical evolution, may go some way to addressing the apparent discrepancy between the observed and calculated accretion rates in the inner disk. We also note here that the disk mass and surface density profile of our model are consistent with observed thermal dust emission from the outer regions of protoplanetary disks and observed accretion rates are mainly derived from optical and UV spectral lines originating from close to the stellar surface (see, e.g., Calvet et al. 2000).

The suppression of turbulence (and thus angular momentum transport) has various further consequences on the disk physical structure and resulting chemistry. A significant source of heating in the disk midplane close to the star is viscous dissipation due to accretion. This can elevate the temperature in this region to $\sim 1000 \mathrm{~K}$, which has implications on the disk chemical structure and resulting line emission. Since this is typically the densest region of the disk, a lot of material is contained within a few AU of the star and thus can have a significant contribution to the disk-integrated line profiles (see, e.g., Carr \& Najita 2008). If accretion is suppressed in this region, this heating cannot occur and so will affect the chemistry and resulting line emission from the inner disk. It is possible that there are alternative sources of ionization in the very hot, dense inner regions of disks, such as thermal ionization, which allow the gas to become magnetorotationally unstable very close to the star (see, e.g., Umebayashi \& Nakano 1988).

We have investigated the effect of turbulent mixing on disk chemical structure (Heinzeller et al. 2011) and find that in the "planet-forming" region, within approximately $10 \mathrm{AU}$ and where the bulk of the infrared line emission originates, turbulent mixing acts to mix material in the vertical direction, dredging material from the disk midplane toward the surface. In fact, we find that our models with turbulent mixing included agree best with current infrared data from disks. In dead zones, the disk flow is essentially laminar and so turbulent mixing within the dead zone itself may be suppressed. This could act to confine a proportion of infrared active molecules to the cold, dense midplane.

Finally, the only negative charge carriers we consider here are electrons and simple negative ions such as $\mathrm{H}^{-}$and $\mathrm{CN}^{-}$. Dust grains and PAHs are also able to capture electrons to form negatively charged grains and PAHs. As discussed previously in Section 2.3, dust grains may be the dominant charge carriers in the disk midplane. Effective accretion depends on the degree of coupling of charged particles to the magnetic field. If PAHs and dust grains are the dominant charge carriers, the relatively large, heavy PAHs and dust grains will increase drag, further opposing accretion. The addition of charged grains is a modification we intend to investigate in future models.

\subsection{Self- and Mutual Shielding of $\mathrm{H}_{2}$ and $\mathrm{CO}$}

The photodissociation of $\mathrm{H}_{2}$ and $\mathrm{CO}$ is dominated by line absorption rather than continuum absorption (see, e.g., Lee et al. 1996); hence, in any atmosphere where there is a significant column density of foreground $\mathrm{H}_{2}$ or $\mathrm{CO}$, there will be a degree of self-shielding against photodissociation dependent on the amount of intervening material. Also, there is an overlap in wavelength ranges over which both molecules absorb leading 


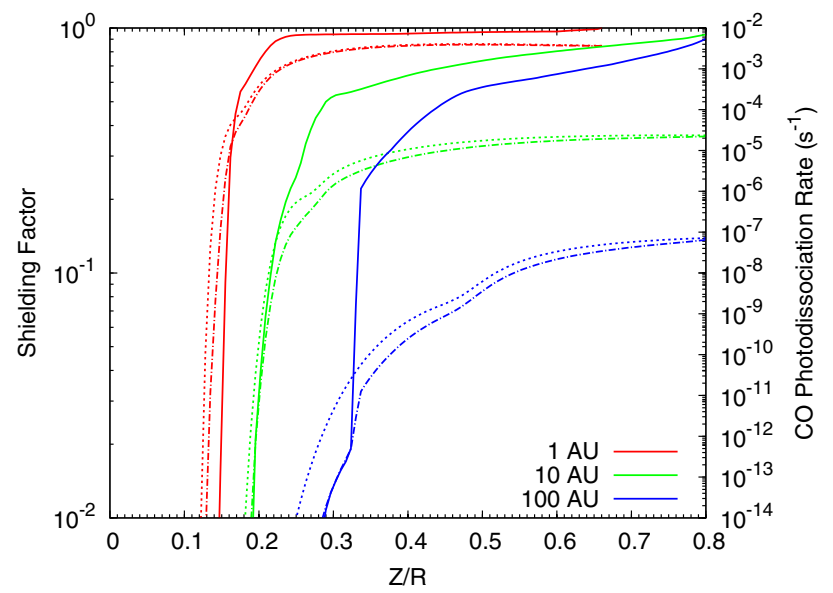

Figure 11. CO shielding factors (solid lines) and photodissociation rates $\left(\mathrm{s}^{-1}\right)$ with (dot-dashed lines) and without (dotted lines) the shielding factors implemented as functions of disk height (scaled by the radius, i.e., $Z / R$ ) at radii of $1 \mathrm{AU}, 10 \mathrm{AU}$, and $100 \mathrm{AU}$.

(A color version of this figure is available in the online journal.)

to a degree of mutual shielding due to the removal of UV flux by the photodissociation of foreground $\mathrm{H}_{2}$.

To date, significant investigation into the self- and mutual shielding of $\mathrm{H}_{2}$ and $\mathrm{CO}$ in photon-dominated regions has been conducted leading to a variety of approximate methods for the computation of self-shielding factors in plane-parallel media (see, e.g., Draine \& Bertoldi 1996; Lee et al. 1996; Visser et al. 2009). In protoplanetary disks, detailed two-dimensional radiative transfer is required since the radiation field has two sources, the central star and the interstellar medium. Also, the addition of self-shielding requires that the abundances of $\mathrm{H}_{2}$ and $\mathrm{CO}$ are known everywhere in the disk at each time step in the calculation simultaneously and this is computationally impossible in a spatially high-resolution model such as this. A compromise often employed in disks is to assume the planeparallel approximation in the vertical direction and calculate the chemical structure from the disk surface downward. In this way, the column density of $\mathrm{H}_{2}$ and $\mathrm{CO}$ from the disk surface to the point of interest can be estimated by extracting out the abundances in the upper layers at a particular time (e.g., $10^{6} \mathrm{yr}$ ). The self-shielding factors calculated for plane-parallel media are then employed in the calculation of the photodissociation rates. However, in the upper disk, where photodestruction is most significant, the direct stellar radiation field is the dominant component so that the column density of material from the star to the point of interest becomes the important parameter opposed to the column density from the disk surface (see Figure 4 in Nomura \& Millar 2005). Also, many self-shielding factors are computed assuming irradiation by the interstellar field only. As we have already discussed, the radiation field in protoplanetary disks will have a very different shape and strength to that found in irradiated interstellar clouds. The computation of such factors also depends on the assumed gas temperature and density since these parameters affect the line shapes and thus absorption cross sections of $\mathrm{H}_{2}$ and $\mathrm{CO}$. In summary, the treatment of self- and mutual shielding in protoplanetary disks is a complex problem and the use of existing approximations to model the selfshielding in disks is problematic as the application of shielding factors computed for irradiated interstellar clouds is debatable.

In the generation of our disk physical model, which originates from Nomura \& Millar (2005), the self-shielding of $\mathrm{H}_{2}$ is included in the calculation (Federman et al. 1979). Here,
Table 5

Unshielded Photodissociation Rates at 1 AU

\begin{tabular}{lccccc}
\hline \hline Species & $\sigma_{\mathrm{Ly}}{ }^{1}$ & $k_{\mathrm{Ly}}$ & $k_{\mathrm{UV}}$ & $k_{\mathrm{Ly}}+k_{\mathrm{UV}}$ & $\left(k_{\mathrm{Ly}}+k_{\mathrm{UV}}\right) / k_{\mathrm{UV}}$ \\
\hline $\mathrm{CO}_{2}$ & $6.1(-20)$ & $5.12(-05)$ & $9.69(-03)$ & $9.74(-03)$ & 1.01 \\
$\mathrm{H}_{2} \mathrm{O}$ & $1.2(-17)$ & $1.01(-02)$ & $1.41(-02)$ & $2.42(-02)$ & 1.72 \\
$\mathrm{HCN}$ & $3.0(-17)$ & $2.52(-02)$ & $1.78(-02)$ & $4.30(-02)$ & 2.52 \\
$\mathrm{OH}$ & $1.8(-18)$ & $1.51(-03)$ & $6.33(-03)$ & $7.84(-03)$ & 1.51 \\
$\mathrm{C}_{2} \mathrm{H}_{2}$ & $4.0(-17)$ & $3.36(-02)$ & $5.16(-02)$ & $8.52(-02)$ & 1.65 \\
$\mathrm{CH}_{4}$ & $1.8(-17)$ & $1.51(-02)$ & $1.44(-02)$ & $2.95(-02)$ & 1.51 \\
$\mathrm{NH}_{3}$ & $1.0(-17)$ & $8.40(-03)$ & $2.56(-02)$ & $3.40(-02)$ & 1.33 \\
\hline
\end{tabular}

Notes. $a(b)$ means $a \times 10^{b}$.

Reference. (1) van Dishoeck et al. 2006.

although we use the calculated $\mathrm{H}$ and $\mathrm{H}_{2}$ abundances from Nomura \& Millar (2005) as initial conditions in our chemical model, the abundances of both species are adjusted to an extent when the full, and vastly more complex, time-dependent chemistry is computed.

In order to quantify the effect that self- and mutual shielding of CO may have in our model, we adopt the method described in Lee et al. (1996) to compute the photodissociation rate of $\mathrm{CO}$ as a function of disk height at a radii of $1 \mathrm{AU}, 10 \mathrm{AU}$, and $100 \mathrm{AU}$ assuming the usual plane-parallel approximation. The results of this calculation are shown in Figure 11. The shielding factors only become significant deep in the atmosphere where the radiation field decreases significantly in strength. We conclude that the addition of self-shielding of $\mathrm{CO}$ using approximation methods has a minor effect on the overall disk structure when compared with the effects of the recalculation of the photorates, taking into consideration the two-dimensional radiation field in the disk. We expect the inclusion of self- and mutual shielding in the radial and vertical directions will lead to an increase in the abundances of $\mathrm{H}_{2}$ and $\mathrm{CO}$ in the outer disk, in particular, since the radial column densities here will be larger than the vertical and the radial stellar radiation field is dominant. We intend to look more closely at the complex issue of self- and mutual shielding of $\mathrm{H}_{2}$ and $\mathrm{CO}$ in disks in future work.

\subsection{On the Effects of Ly $\alpha$ Radiation}

In Section 2.2.1 we discussed how we included Ly $\alpha$ radiation in the calculation of the wavelength-integrated flux yet neglected it in the determination of the wavelength-dependent UV spectrum throughout the disk. This was due to the difficulty in treating $\operatorname{Ly} \alpha$ scattering in protoplanetary disks, an issue only recently addressed in work by Bethell \& Bergin (2011). Here, we consider the effects of the addition of $\operatorname{Ly} \alpha$ on the calculated photodissociation rates of several molecules.

We calculate the photodissociation rates using the stellar spectrum displayed in the left-hand panel of Figure 1, which is the unshielded UV photon flux at the disk surface at a radius of $1 \mathrm{AU}$. Note that this spectrum is different from that used to calculate the photorates displayed in Figure 6. In our full disk model, we assume that the stellar radiation has traveled through, and thus been attenuated by, material along the line of sight between the star and the disk surface.

We calculate the unshielded photorates with and without $\operatorname{Ly} \alpha$, using the calculated Ly $\alpha$ cross sections from Table 1 in van Dishoeck et al. (2006). Our results are presented in Table 5. In our determination of the photorate at the Ly $\alpha$ wavelength, we assume that the cross section is constant across the line, determine the rate due to $\operatorname{Ly} \alpha$ only, and add that to the rate calculated by integrating over the background UV field (see 
Equations (3) and (4)). The integrated photon flux over the Ly $\alpha$ line is $8.4 \times 10^{14}$ photons $\mathrm{cm}^{-2} \mathrm{~s}^{-1}$.

Note that the photodissociation cross section of most molecules at the Ly $\alpha$ wavelength is generally lower than at other wavelengths, and hence we find that the inclusion of Ly $\alpha$ increases the photodissociation rates by no more than a factor of 2.52 (as determined for $\mathrm{HCN}$ ). In general, the enhancement is less than a factor of two, which is well within the uncertainties in both the UV spectrum and the photo cross sections. Even though the UV field is weaker at wavelengths other than the Ly $\alpha$ wavelength (see Figure 1) the overall contribution of Ly $\alpha$ photons to the photodissociation rates is around the same order of magnitude as the contribution by the background UV photons, i.e., we do not see the order-of-magnitude difference in the photorates when the Ly $\alpha$ photon flux is included in the integrated UV field and the interstellar rates scaled by this flux (see Figure 5). This result is in agreement with the work of Fogel et al. (2011) who find that the inclusion of Ly $\alpha$ has an effect on the chemistry only when grain settling is included in their model.

\subsection{Comparison with Other Models}

A full two-dimensional calculation of the UV radiative transfer in disks was achieved by van Zadelhoff et al. (2003). They coupled the UV radiation field and photochemistry and applied their chemical model to the established protoplanetary disk model of D'Alessio et al. (1999). In this earlier work, they calculate the photorates adopting difference shapes for the stellar UV field and come to a similar conclusion as ourselves, i.e., the abundances of molecules are sensitive to the shape of the adopted stellar UV spectrum. They also find at a radius of $105 \mathrm{AU}$ in their model that the photodissociation rates of $\mathrm{C}_{2} \mathrm{H}$ and $\mathrm{H}_{2} \mathrm{CO}$ are generally underestimated when the UV stellar spectrum is approximated by a scaling of the ISRF.

Early investigations into the calculation of the X-ray ionization in disks include the work of Aikawa \& Herbst (1999, 2001). Aikawa \& Herbst (1999) adopt a power-law approximation for the X-ray absorption cross section similar to that used in Paper I (Maloney et al. 1996). Aikawa \& Herbst (2001) perform a calculation similar to that adopted here, where the explicit elemental composition and absorption cross section are taken into account as is the direct X-ray ionization of elements. Although their underlying disk physical model is analytical in nature and different from ours in that the X-rays are included in purely a chemical sense, i.e., X-ray heating of the disk is not taken into account, they too find little difference between results using either method. More recent models based on the work of Aikawa \& Herbst (2001) (e.g., Woods \& Willacy 2009) have adopted this method of calculating the X-ray ionization rates.

Aikawa \& Nomura (2006) include spectrum-dependent photochemistry looking at the effect of grain growth on disk chemical structure, although they neglect the effects of X-rays. The physical model used in their work is based on the model of Nomura \& Millar (2005) where a full two-dimensional calculation of the radiative transfer allowed the determination of the UV spectrum (including UV excess emission from the star) everywhere in the disk. We currently have in preparation a paper where we look at the effects of grain growth in a protoplanetary disk in which X-rays are included (C. Walsh et al. 2012, in preparation).

The disk model of Woitke et al. (2009), "ProDiMo," has recently been updated with spectrum-dependent photochemistry (Kamp et al. 2010) and X-ray ionization (Aresu et al. 2011) with the methods used and cross-sectional data adopted similar to that used here. The recent work by Vasyunin et al. (2011) now includes wavelength-dependent photochemistry, updating their previous model (Semenov et al. 2005; Vasyunin et al. 2008) to investigate the effects of grain settling on the disk chemistry. Also, Fogel et al. (2011) have included photochemistry investigating the effect of $\mathrm{Ly} \alpha$ radiation on the disk chemical structure. They find, in models in which dust settling is included, those species with significant photodissociation cross sections around the Ly $\alpha$ line are particularly affected, generally leading to a reduction in column density for species such as $\mathrm{HCN}, \mathrm{NH}_{3}$, and $\mathrm{CH}_{4}$. This effect is significantly less in models where they assume that the dust is well mixed with the gas, an assumption we adopt in our work.

\subsection{Comparison with Observation}

The observation of molecular line emission from protoplanetary disks remains a challenge due to the small angular size of these objects and the limitations of existing (sub)millimeter observing facilities. Ideally, to directly compare our results with observations, full radiative transfer should be performed and disk-averaged line profiles and intensities calculated to compare with those measured by telescopes.

Our model is not specific to any particular source and we have adopted the star-disk parameters of a typical T Tauri star (Hartmann \& Kenyon 1996). However, since we adopt the $\mathrm{X}$-ray spectrum and UV excess observed from the T Tauri star, TW Hya, we compare our calculated column densities with those derived from molecular line observations (single dish and interferometric) of this source. TW Hya is a well-studied, relatively old $(\sim 10 \mathrm{Myr})$, nearby $(51 \pm 4 \mathrm{pc})$, almost faceon $\left(\lesssim 10^{\circ}\right)$, isolated classical T Tauri star (see, e.g., Kastner et al. 1997; Webb et al. 1999; Krist et al. 2000; Mamajek 2005). Observations of continuum dust emission from TW Hya suggest it has a cleared inner hole, possibly caused by a forming planet, and a truncated outer disk with radius $\approx 200 \mathrm{AU}$ (Calvet et al. 2002; Setiawan et al. 2008; Akeson et al. 2011).

Rotational line emission at (sub)millimeter wavelengths from several molecules has been observed in TW Hya including $\mathrm{CO}$, $\mathrm{CN}, \mathrm{HCO}^{+}, \mathrm{HCN}$, as well as the isotopologues, ${ }^{13} \mathrm{CO}, \mathrm{H}^{13} \mathrm{CO}^{+}$, $\mathrm{DCO}^{+}$, and DCN (Kastner et al. 1997; van Zadelhoff et al. 2001; van Dishoeck et al. 2003; Wilner et al. 2003; Qi et al. 2004, 2006, 2008; Thi et al. 2004). Molecular line emission has been spatially mapped by Qi et al. (2004, 2006, 2008) using the SMA and by Wilner et al. (2003) using the Australia Telescope Compact Array. More recently, Hogerheijde et al. (2011) report the detection of the ground-state emission lines of both spin isomers of $\mathrm{H}_{2} \mathrm{O}$ in TW Hya observed using the HIFI on the Herschel Space Observatory. In addition to (sub)millimeter data, Najita et al. (2010) present detections of $\mathrm{OH}, \mathrm{CO}_{2}, \mathrm{HCO}^{+}$, and possibly $\mathrm{CH}_{3}$, at mid-infrared wavelengths using the IRS on board the Spitzer Space Telescope.

Thi et al. (2004) and Qi et al. (2008) each use a method to derive the column density of each molecule using their observed line profiles. Thi et al. (2004) assume that the emitting region has a typical density and temperature, constrained by line ratios in a single molecule, and assuming that the energy levels are thermalized (i.e., they assume local thermodynamic equilibrium). The optical depth can be estimated using line ratios of the same transition in two isotopologues assuming the same excitation temperature. They list their beam-averaged column densities for TW Hya assuming a disk radius of $165 \mathrm{AU}$ and an excitation temperature of $25 \mathrm{~K}$. We reproduce their calculated values in Table 6. Qi et al. (2008) constrain their estimated 
Table 6

TW Hya Observed Column Densities

\begin{tabular}{lr}
\hline \hline Species & $\begin{array}{r}N \\
\left(\mathrm{~cm}^{-2}\right)\end{array}$ \\
\hline Beam-averaged values from Thi et al. $(2004)$
\end{tabular}

column densities between $10 \mathrm{AU}$ and 100 AU by assuming a simple power-law distribution for the surface density of the disk and fitting the vertical extent and abundance of each molecule to match the observed line profiles using a $\chi^{2}$ method. Again, we list their fitted values at a radius of $\approx 100 \mathrm{AU}$ in Table 6 .

Comparing our calculated column densities at a radius of $100 \mathrm{AU}$ (listed in Table 4) with those derived from observation, we see our value for $\mathrm{CO}$ is more than two orders of magnitude larger than the value from Thi et al. (2004). Comparing the values for $\mathrm{HCO}^{+}$and $\mathrm{HCN}$, we see good agreement between the column densities from both authors, however, our calculated column densities for both species are around one order of magnitude larger than the observed values. Looking at $\mathrm{CN}$, we see the same behavior with our model predicting a value between 2 and $5 \times 10^{14} \mathrm{~cm}^{2}$ compared with the observed value of $6.6 \times 10^{13} \mathrm{~cm}^{2}$.

Unfortunately, Hogerheijde et al. (2011) do not report their results in terms of column density, however, they do interpret their results using the detailed disk model of Woitke et al. (2009) calculating a total water vapor mass of $7.3 \times 10^{24} \mathrm{~g}$ in a disk with total mass $1.9 \times 10^{-2} M_{\odot}$. This corresponds to a disk-averaged water vapor fractional abundance of $\approx 2 \times$ $10^{-8}$. Taking our modeled column densities of $\mathrm{H}_{2}$ and $\mathrm{H}_{2} \mathrm{O}$ at $100 \mathrm{AU}$ for model $\mathrm{XR}+\mathrm{UV}$-new, we find a column-averaged fractional abundance of $7 \times 10^{-8}$, which compares well with the disk-averaged value. Hogerheijde et al. (2011) report that their observations are optically thin; hence the line emission is tracing the entire column density of water throughout the vertical extent of the disk. Also, Najita et al. (2010) do not convert their line observations to physical quantities such as column density or fractional abundance since they plan to discuss their results for $\mathrm{OH}, \mathrm{HCO}^{+}$, and $\mathrm{CO}_{2}$ in more detail in a future publication. They do mention their apparent anomalous result for TW Hya which is their non-detection of $\mathrm{H}_{2} \mathrm{O}, \mathrm{C}_{2} \mathrm{H}_{2}$, and $\mathrm{HCN}$ line emission, in contrast to results for most classical T Tauri stars observed using IRS (Carr \& Najita 2011; Salyk et al. 2011). One explanation they give is that TW Hya is unusual in that is apparently at a more advanced stage than its accretion rate suggests, i.e., TW Hya is not an archetypical classical T Tauri star.

Why is our model overestimating the column densities, especially those observed with ground-based facilities? One reason is that our disk is not meant to be specific to TW Hya even though we adopt the X-ray and UV spectrum of this source in our model. Another reason is that our column densities involve integration over the full vertical extent of the disk from the lower disk surface to the upper disk surface. In reality, the disk is likely to be optically thick, for ${ }^{12} \mathrm{CO}$ and $\mathrm{H}^{12} \mathrm{CO}^{+}$emission particularly, and so the observed line emission is only tracing a fraction of the total column density. A third reason is that approximations for the disk chemical and physical structure are used for generating observed column densities rather than a detailed model which allows for vertical and radial structure in the disk density and temperature and resulting molecular abundances.

As stated previously, in order to determine how accurate our model is, one must perform full radiative transfer and compare our modeled line profiles with those observed.

\section{SUMMARY}

We have investigated the effects of photochemistry and $\mathrm{X}$-ray ionization on the chemical structure of a protoplanetary disk surrounding a typical T Tauri star. We used a high-resolution complex physical model which takes into account irradiation by both the central star and the interstellar medium. We compiled a comprehensive chemical network including a large gas-phase reaction set extracted from the UDfA (Rate06), gas-grain interactions (accretion, thermal desorption, cosmic-ray-induced desorption, and photodesorption), and a grain-surface network.

In previous work, we presented results from a model in which we approximated the photorates in the disk by scaling those in Rate06 (which assume the ISRF) by the wavelength-integrated UV flux at each point. The X-ray ionization rate everywhere was also approximated using a power law to describe the energydependent cross section for ionization. Here, we recalculated both the photorates and the X-ray ionization rate, in the first case taking into account the unique UV wavelength spectrum at each point, and in the second, the unique X-ray energy spectrum and the explicit elemental composition of our gas. We also added the direct X-ray ionization of elements.

We found that the recalculation of the photochemistry has a much larger effect on the disk chemical structure than that for the $\mathrm{X}$-ray ionization. Concentrating on those molecules which have been observed or searched for in disks at either (sub)millimeter or infrared wavelengths, we find that the species most sensitive to the photochemistry are $\mathrm{OH}, \mathrm{HCO}^{+}, \mathrm{N}_{2} \mathrm{H}^{+}, \mathrm{H}_{2} \mathrm{O}, \mathrm{CO}_{2}$, and methanol. We also find that the radicals, $\mathrm{CN}$ and $\mathrm{C}_{2} \mathrm{H}$, are also affected, although not to the same extent as those listed above. The recalculation of the photochemistry affects each molecule in a different manner, indicating the inherent nonlinearity of the chemistry. Molecules affected throughout the disk extent include $\mathrm{HCO}^{+}, \mathrm{N}_{2} \mathrm{H}^{+}$, and $\mathrm{OH}$, i.e., predominantly molecular ions and radicals. Water and methanol are mainly affected beyond a radius of around $1 \mathrm{AU}$, whereas $\mathrm{CO}_{2}$ is altered beyond approximately $10 \mathrm{AU}$. The differences in the behavior of the saturated molecules can be attributed to the location of the molecular layer in each case, with methanol and water residing in a layer higher in the disk than $\mathrm{CO}_{2}$ which in turn is linked to the desorption temperature of both molecules, with water more strongly bound to the grain surfaces than carbon dioxide (see Table 2).

In general, we find that the change in the photochemistry leads to a depletion of molecules in the inner disk (within $1 \mathrm{AU}$ ) and an enhancement in the outer disk (beyond $1 \mathrm{AU}$ ). We conclude that, in the outer disk, our original approximation was overestimating the photorates, whereas, in the inner disk, where the stellar irradiation is strongest, we were underestimating the photorates. We should note that the photochemistry also indirectly affects the abundances of molecules via gas-phase chemistry. In models where we recalculate the photorates, we find differences in the location of the $\mathrm{H} / \mathrm{H}_{2}$ and $\mathrm{C}^{+} / \mathrm{C}$ transition regions with both ratios generally decreasing in the molecular layer in models where we recalculate the photochemistry. Neutral molecules such as water and $\mathrm{CO}_{2}$ are effectively destroyed by reaction 
with $\mathrm{C}^{+}$and in regions of high temperature by reaction with $\mathrm{H}$ atoms. Also, the molecular ions, $\mathrm{HCO}^{+}$and $\mathrm{N}_{2} \mathrm{H}^{+}$, are efficiently destroyed via dissociative electron recombination.

Concerning the X-ray ionization rate, we find that the only molecule especially affected is $\mathrm{N}_{2} \mathrm{H}^{+}$. Residing in a different region of the disk to those molecules discussed thus far, we see the effect of X-ray ionization in the outer disk, in particular, where the abundance and distribution of $\mathrm{N}_{2} \mathrm{H}^{+}$are decreased. Since the chemistry of $\mathrm{N}_{2} \mathrm{H}^{+}$is linked closely to the ionization of molecular hydrogen in the disk, this is unsurprising; however, one conclusion we can draw is that observations of $\mathrm{N}_{2} \mathrm{H}^{+}$emission from disks in conjunction with observations of those molecules unaffected by X-rays, e.g., CO, could give us information on the effects of X-ray ionization in the outer regions of protoplanetary disks around classical T Tauri stars.

The ionization fraction in the disk is affected only marginally by the recalculation of the photochemical and X-ray ionization rates. We find that photochemistry affects the electron abundance in the disk surface whereas X-rays affect the disk midplane and outer disk region, since $\mathrm{X}$-rays are able to penetrate the disk more effectively than UV photons.

A calculation of the magnetic Reynolds number and ambipolar diffusion parameter everywhere in our disk allowed us to determine the location of a possible significant dead zone in the disk midplane extending to $\approx 200 \mathrm{AU}$ where accretion will likely be suppressed. This has implications on both the physical and chemical structure since a significant source of heating in the disk midplane close to the star is viscous dissipation due to accretion flow. Our calculations suggest that the accretion rate in the outer regions of our disk model is only $\approx 70 \%$ of that used to determine the disk physical structure. In the inner disk, this value falls to $\approx 1 \%$; however, we have neglected alternative sources of ionization which may dominate in the inner disk, e.g., the thermal ionization of alkali models, which should be considered in future models.

We conclude that a wavelength-dependent treatment of the photochemistry in protoplanetary disks is necessary since it significantly affects the chemical structure. We also determine that existing approximations of the X-ray ionization rate in such objects are sufficient, affecting only the abundance and distribution of $\mathrm{N}_{2} \mathrm{H}^{+}$. In order to directly compare our model results with observation and to determine the effects of the various chemical processes investigated in our work on observable line emission we have computed the radiative transfer and modeled molecular line emission from the disk both at the resolution of existing facilities and at the expected resolution of ALMA. We report the results from these calculations in a subsequent publication (C. Walsh et al. 2012, in preparation).

We thank an anonymous referee for comments which greatly improved the content and scope of the manuscript. C.W. acknowledges DEL for a studentship and JSPS for the award of a short-term fellowship to conduct research in Japan. H.N. acknowledges the JGC-S Scholarship Foundation, the Grant-in-Aid for Scientific Research 21740137 and the Global COE Program "The Next Generation of Physics, Spun from Universality and Emergence" from MEXT, Japan. Astrophysics at QUB is supported by a grant from the STFC.

\section{REFERENCES}

Aikawa, Y., \& Herbst, E. 1999, A\&A, 351, 233

Aikawa, Y., \& Herbst, E. 2001, A\&A, 371, 1107
Aikawa, Y., Momose, M., Thi, W.-F., et al. 2003, PASJ, 55, 11

Aikawa, Y., \& Nomura, H. 2006, ApJ, 642, 1152

Akeson, R. L., Millan-Gabet, R., Ciardi, D. R., et al. 2011, ApJ, 728, 96

Aresu, G., Kamp, I., Meijerink, R., et al. 2011, A\&A, 526, A163

Armitage, P. J., Livio, M., \& Pringle, J. E. 2001, MNRAS, 324, 705

Bai, X.-N. 2011, ApJ, 739, 50

Bai, X.-N., \& Stone, J. M. 2011, ApJ, 736, 144

Balbus, S. A., \& Hawley, J. F. 1991, ApJ, 376, 214

Bergin, E., Calvet, N., D’Alessio, P., \& Herczeg, G. J. 2003, ApJ, 591, L159

Bergin, E. A., Aikawa, Y., Blake, G. A., \& van Dishoeck, E. F. 2007, in Protostars and Planets V, ed. B. Reipurth, D. Jewitt, \& K. Keil (Tuscon, AZ: Univ. Arizona Press), 751

Bethell, T. J., \& Bergin, E. A. 2011, ApJ, 739, 78

Brown, W. A., \& Bolina, A. S. 2007, MNRAS, 374, 1006

Calvet, N., D’Alessio, P., Hartmann, L., et al. 2002, ApJ, 568, 1008

Calvet, N., Hartmann, L., \& Strom, S. E. 2000, in Protostars and Planets IV, ed. V. Mannings, A. P. Boss, \& S. S. Russell (Tuscon, AZ: Univ. Arizona Press), 377

Carr, J. S., \& Najita, J. R. 2008, Science, 319, 1504

Carr, J. S., \& Najita, J. R. 2011, ApJ, 733, 102

Chiang, E., \& Murray-Clay, R. 2007, Nat. Phys., 3, 604

Chiang, E. I., Joung, M. K., Creech-Eakman, M. J., et al. 2001, ApJ, 547, 1077

Ciesla, F. J. 2007, ApJ, 654, L159

Creech-Eakman, M. J., Chiang, E. I., Joung, R. M. K., Blake, G. A., \& van Dishoeck, E. F. 2002, A\&A, 385, 546

D’Alessio, P., Calvet, N., Hartmann, L., Franco-Hernández, R., \& Servín, H. 2006, ApJ, 638, 314

D’Alessio, P., Calvet, N., Hartmann, L., Lizano, S., \& Cantó, J. 1999, ApJ, 527. 893

Draine, B. T. 1978 , ApJS, 36, 595

Draine, B. T., \& Bertoldi, F. 1996, ApJ, 468, 269

Dullemond, C. P., \& Dominik, C. 2004, A\&A, 421, 1075

Dutrey, A., Guilloteau, S., \& Guélin, M. 1997, A\&A, 317, L55

Edridge, J. L. 2010, PhD thesis, Univ. College London

Federman, S. R., Glassgold, A. E., \& Kwan, J. 1979, ApJ, 227, 466

Fogel, J. K. J., Bethell, T. J., Bergin, E. A., Calvet, N., \& Semenov, D. 2011, ApJ, 726,29

Fromang, S., \& Papaloizou, J. 2006, A\&A, 452, 751

Fromang, S., Terquem, C., \& Balbus, S. A. 2002, MNRAS, 329, 18

Fuente, A., Cernicharo, J., Agúndez, M., et al. 2010, A\&A, 524, A19

Gammie, C. F. 1996, ApJ, 457, 355

Glassgold, A. E., Meijerink, R., \& Najita, J. R. 2009, ApJ, 701, 142

Glassgold, A. E., Najita, J., \& Igea, J. 1997, ApJ, 480, 344

Graedel, T. E., Langer, W. D., \& Frerking, M. A. 1982, ApJS, 48, 321

Hartmann, L., \& Kenyon, S. J. 1996, ARA\&A, 34, 207

Hasegawa, T. I., \& Herbst, E. 1993, MNRAS, 261, 83

Hasegawa, T. I., Herbst, E., \& Leung, C. M. 1992, ApJS, 82, 167

Hawley, J. F., \& Stone, J. M. 1998, ApJ, 501, 758

Heinzeller, D., Nomura, H., Walsh, C., \& Millar, T. J. 2011, ApJ, 731, 115

Herbig, G. H. 1977, ApJ, 217, 693

Herbig, G. H., \& Goodrich, R. W. 1986, ApJ, 309, 294

Herczeg, G. J., Linsky, J. L., Valenti, J. A., Johns-Krull, C. M., \& Wood, B. E. 2002, ApJ, 572, 310

Herrero, V. J., Gálvez, Ó, Belén, M., \& Escribano, R. 2010, Phys. Chem. Chem. Phys., 12, 3164

Hogerheijde, M. R., Bergin, E. A., Brinch, C., et al. 2011, Science, 334, 338

Ilgner, M., \& Nelson, R. P. 2006, A\&A, 445, 205

Jansen, D. J., Spaans, M., Hogerheijde, M. R., \& van Dishoeck, E. F. 1995a, A\&A, 303, 541

Jansen, D. J., van Dishoeck, E. F., Black, J. H., Spaans, M., \& Sosin, C. 1995b, A\&A, 302, 223

Kamp, I., Tilling, I., Woitke, P., Thi, W.-F., \& Hogerheijde, M. 2010, A\&A, 510, A18

Kastner, J. H., Huenemoerder, D. P., Schulz, N. S., Canizares, C. R., \& Weintraub, D. A. 2002, ApJ, 567, 434

Kastner, J. H., Zuckerman, B., Weintraub, D. A., \& Forveille, T. 1997, Science, 277,67

Krist, J. E., Stapelfeldt, K. R., Ménard, F., Padgett, D. L., \& Burrows, C. J. 2000, ApJ, 538, 793

Kunz, M. W., \& Balbus, S. A. 2004, MNRAS, 348, 355

Lahuis, F., van Dishoeck, E. F., Boogert, A. C. A., et al. 2006, ApJ, 636, L145

Lee, H.-H., Herbst, E., Pineau des Forêts, G., Roueff, E., \& Le Bourlot, J. 1996, A\&A, 311, 690

Léger, A., Jura, M., \& Omont, A. 1985, A\&A, 144, 147

Liedahl, D. A., Osterheld, A. L., \& Goldstein, W. H. 1995, ApJ, 438, L115

Lynden-Bell, D., \& Pringle, J. E. 1974, MNRAS, 168, 603

Maloney, P. R., Hollenbach, D. J., \& Tielens, A. G. G. M. 1996, ApJ, 466, 561 
Mamajek, E. E. 2005, ApJ, 634, 1385

Najita, J. R., Carr, J. S., Strom, S. E., et al. 2010, ApJ, 712, 274

Nomura, H., Aikawa, Y., Tsujimoto, M., Nakagawa, Y., \& Millar, T. J. 2007, ApJ, 661,334

Nomura, H., \& Millar, T. J. 2005, A\&A, 438, 923

Öberg, K. I., Fuchs, G. W., Awad, Z., et al. 2007, ApJ, 662, L23

Öberg, K. I., Garrod, R. T., van Dishoeck, E. F., \& Linnartz, H. 2009a, A\&A, 504,891

Öberg, K. I., Linnartz, H., Visser, R., \& van Dishoeck, E. F. 2009b, ApJ, 693 , 1209

Öberg, K. I., Qi, C., Fogel, J. K., et al. 2010, ApJ, 720, 480

Öberg, K. I., van Broekhuizen, F., Fraser, H. J., et al. 2005, ApJ, 621, L33

Öberg, K. I., van Dishoeck, E. F., \& Linnartz, H. 2009c, A\&A, 496, 281

Perez-Becker, D., \& Chiang, E. 2011, ApJ, 735, 8

Pontoppidan, K. M., Salyk, C., Blake, G. A., et al. 2010, ApJ, 720, 887

Pringle, J. E. 1981, ARA\&A, 19, 137

Qi, C., Ho, P. T. P., Wilner, D. J., et al. 2004, ApJ, 616, L11

Qi, C., Wilner, D. J., Aikawa, Y., Blake, G. A., \& Hogerheijde, M. R. 2008, ApJ, 681,1396

Qi, C., Wilner, D. J., Calvet, N., et al. 2006, ApJ, 636, L157

Salyk, C., Pontoppidan, K. M., Blake, G. A., Najita, J. R., \& Carr, J. S. 2011, ApJ, 731,130

Salyk, C., Pontoppidan, K. M., Blake, G. A., et al. 2008, ApJ, 676, L49

Sano, T., Miyama, S. M., Umebayashi, T., \& Nakano, T. 2000, ApJ, 543, 486

Sano, T., \& Stone, J. M. 2002, ApJ, 577, 534

Schegerer, A. A., \& Wolf, S. 2010, A\&A, 517, A87

Semenov, D., Pavyluchenkov, Ya., Schreyer, K., et al. 2005, ApJ, 621, 853

Setiawan, J., Henning, Th., Launhardt, R., et al. 2008, Nature, 451, 38

Shakura, N. I., \& Sunyaev, R. A. 1973, A\&A, 24, 337

Terada, H., Tokunaga, A. T., Kobayashi, N., et al. 2007, ApJ, 667, 303

Thi, W.-F., van Zadelhoff, G.-J., \& van Dishoeck, E. F. 2004, A\&A, 425, 955
Turner, N. J., Carballido, A., \& Sano, T. 2010, ApJ, 708, 188

Umebayashi, T., \& Nakano, T. 1988, Prog. Theor. Phys. Suppl., 96, 151 van Dishoeck, E. F. 1987, in IAU Symp. 120, Astrochemistry, ed. M. S. Vardya \& S. P. Tarafdar (Dordrecht: Kluwer), 51

van Dishoeck, E. F. 1988, in Rate Coefficients in Astrochemistry, ed. T. J. Millar \& D. A. Williams (Dordrecht: Kluwer), 49

van Dishoeck, E. F., Jonkheid, B., \& van Hemert, M. C. 2006, Faraday Discuss., 133,231

van Dishoeck, E. F., Thi, W.-F., \& van Zadelhoff, G.-J. 2003, A\&A, 400, L1 van Zadelhoff, G.-J., Aikawa, Y., Hogerheijde, M. R., \& van Dishoeck, E. F. 2003, A\&A, 397, 789

van Zadelhoff, G.-J., van Dishoeck, E. F., Thi, W.-F., \& Blake, G. A. 2001, A\&A, 377,566

Vasyunin, A. I., Semenov, D., Henning, Th., et al. 2008, ApJ, 672, 629

Vasyunin, A. I., Wiebe, D. S., Birnstiel, T., et al. 2011, ApJ, 727, 76

Verner, D. A., Yakovlev, D. G., Band, I. M., \& Trzhaskovskaya, M. B. 1993, At. Data Nucl. Data Tables, 55, 233

Visser, R., van Dishoeck, E. F., \& Black, J. H. 2009, A\&A, 503, 323

Vorobyov, E. I., \& Basu, S. 2006, ApJ, 650, 956

Walsh, C., Millar, T. J., \& Nomura, H. 2010, ApJ, 722, 1607

Webb, R. A., Zuckerman, B., Platais, I., et al. 1999, ApJ, 512, L63

Weingartner, J. C., \& Draine, B. T. 2001, ApJ, 548, 296

Westley, M. S., Baragiola, R. A., Johnson, R. E., \& Baratta, G. A. 1995, Planet. Space Sci., 43, 1311

Willacy, K. 2007, ApJ, 660, 441

Willacy, K., \& Langer, W. D. 2000, ApJ, 544, 903

Wilner, D. J., Bourke, T. L., Wright, C. M., et al. 2003, ApJ, 596, 597

Woitke, P., Kamp, I., \& Thi, W.-F. 2009, A\&A, 501, 383

Woodall, J., Agúndez, M., Markwick-Kemper, A. J., \& Millar, T. J. 2007, A\&A, 466, 1197

Woods, P. M., \& Willacy, K. 2009, ApJ, 693, 1360 